\title{
The hyperbolic wavelet transform: an efficient tool for multifractal analysis of anisotropic fields
}

\author{
Patrice Abry, Marianne Clausel, Stéphane Jaffard, \\ Stéphane G. Roux and Béatrice Vedel
}

\begin{abstract}
Global and local regularity of functions in anisotropic function spaces is analyzed in the common framework provided by hyperbolic wavelet bases. Local and directional regularity features are characterized by means of global quantities derived from the coefficients of hyperbolic wavelet decompositions. A multifractal analysis is introduced, that jointly accounts for scale invariance and anisotropy, and its properties are investigated.
\end{abstract}

\section{Introduction}

Natural images often display various forms of anisotropy. For a wide range of applications, anisotropy has been quantified through regularity characteristics and features that differ strongly when measured in different directions. This is, for instance, the case in medical imaging (osteoporosis, muscular tissues, mammographies, etc.), see e.g. [13] and [14], hydrology [41], fracture surfaces analysis [20]. For such images, key issues are, first, to describe the anisotropy of the texture, and then to define regularity anisotropy parameters that can be measured via numerical procedures and further developed into e.g., classification schemes. This requires the design of a mathematical framework that allows to define and estimate these parameters. Such a program can be split into several questions, some of them having already been either solved or, at least, partially addressed. We start by briefly sketching this program.

Important examples of anisotropic self-similar fields governed by two parameters (an anisotropy matrix and a self-similarity index) have been introduced and studied by H. Biermé, M. Meerschaert and H. Scheffler in [13], as a model relevant for describing osteoporosis.

Mathematics Subject Classification (2010): Primary 46E35; Secondary 42C40.

Keywords: Hyperbolic wavelet analysis, anisotropic Besov spaces, pointwise Hölder regularity, anisotropic multifractal analysis. 
In [19], two of the authors (M. Clausel and B. Vedel) addressed the question of defining in a proper way the concept of the anisotropy of an image in relation to its global regularity. In particular they showed that the two parameters characterizing the above mentioned random fields can be recovered even in the absence of a priori knowledge of the characteristics of the model, by studying the global smoothness properties of the process. This result indicated that some of the properties characterizing anisotropy are revealed by the regularity of the sample paths when analyzed with functional spaces well adapted to anisotropy, namely anisotropic Besov spaces, which extend (isotropic) Besov spaces (see Section 2.1 for a definition). This first result illuminated the key role that such spaces might play in the analysis of random anisotropic textures. For example, in two variables, such spaces present the following anisotropy property. They are invariant after rescaling by $\lambda^{\alpha}$ along the $x$-axis and by $\lambda^{2-\alpha}$ along the $y$-axis, where $\alpha$ is the anisotropy parameter.

Note that this point of view follows, in the isotropic setting, ideas that are common in multifractal analysis, where global function space regularity yields parameters pertinent to classification; see [1] and references therein. This also explains the choice of Besov spaces instead of Sobolev spaces, which (in addition to a simpler wavelet characterization) allows to use Lebesgue integrability indices $p \in(0,1)$, therefore yielding a larger range of classification indices.

Note that, in the isotropic setting, wavelet bases provide an efficient tool for measuring smoothness in a large range of functional spaces (see [36] for details). For a given anisotropy parameter, anisotropic wavelets such as curvelets, bandelets, contourlets, shearlets, ridgelets, or wedgelets have been introduced (see e.g. [32] for a thorough review of these representation systems and a comparison of their properties for image processing). Natural criteria for choosing between these alternatives are, on the mathematical side, that these variations on isotropic wavelets provide bases for the corresponding anisotropic spaces, and, on the applied side, that practically tractable procedures can be devised and implemented to permit the characterization of real-world data according to these function spaces.

On the mathematical side, many authors have addressed the problem of obtaining bases of a given anisotropic Besov space, and have proposed different solutions, depending on the precise definition of anisotropic spaces they have used; see e.g. [10], [9], [21], [31], [30], [48], and [49], and also the recent papers [26] by G. Garrigós and A. Tabacco, and [29] by D. Haroske and E. Tamási, which contain numerous references on the subject. Note also that, in several cases, the study was restricted to a specific anisotropy parameter; typically, parabolic anisotropy, where a contraction by $\lambda$ in one direction is associated with a contraction by $\lambda^{2}$ along the orthogonal direction, (see [35], [39], and [43], and references therein, in particular for applications to directional regularity). The corresponding dictionaries are in that case curvelets or contourlets (corresponding to the Hart Smith decomposition in the continuous setting; see [46]). This choice was motivated from application to PDEs where this specific anisotropy has physical relevance (see e.g. [18] and [28]), where curvelets and ridgelets are used for the study of Fourier integral operators, with applications to the wave equation); however, it is no longer justified when 
dealing with images, where no particular form of anisotropy can be postulated a priori. Actually, except perhaps in very specific applications, the particular type of anisotropy present in an image is not known a priori, and determining it out is part of the problem. Therefore a key practical issue for us is that we must deal simultaneously with the whole collection of anisotropic Besov spaces, for all possible anisotropies. This raises the mathematical question of finding specific bases which could serve as a common "dictionary" for all of these spaces simultaneously, and obtaining simple characterizations of those bases in this "dictionary". Clearly, the dictionary used should display all possible types of anisotropy; among the variants of directional wavelets that are not tailored to a specific anisotropy, two play a prominent role because of their numerical simplicity (as tensor products of 1-D wavelets) and their good functional properties:

- One is the collection of anisotropic Triebel bases; see [49]. Each basis is constructed from the standard wavelet through a multiresolution procedure, tailored to a specific anisotropy. Simple characterizations of Besov spaces (with the same anisotropy) have been given for this system. Such characterizations can thus be used as building blocks for constructing a multifractal formalism; see [6] for first results in this direction, and also Section 3. Triebel bases provide a powerful tool for deducing results on a given anisotropic Besov space; in particular, they make it possible to show that these spaces are isomorphic to the corresponding isotropic Besov spaces. Furthermore, some results such as embeddings or profiles of Besov characteristics can be obtained, via the transference method proposed by H. Triebel. However, knowledge of the expansion of a function in one basis gives neither information about its expansion in another basis nor about whether it belongs to other anisotropic Besov spaces. Therefore, this tool does not seem to help understand the link between different forms of anisotropy, in terms of function spaces, for example.

In order to deal with situations where no anisotropy is prescribed, a natural idea consists in using the union of these bases, for all possible anisotropies. One easily checks that this dictionary is too redundant to constitute a frame. However, because many of their elements coincide (and therefore need not be duplicated), one cannot exclude the possibility that the union of these bases could nonetheless prove an efficient tool for analyzing anisotropic fields with no a priori anisotropy.

- Another possible decomposition system is given by hyperbolic wavelets, introduced in various settings under various denominations (standard, rectangular or hyperbolic wavelet analysis), notably in image coding (see [51]), numerical analysis (see [11], [12]), and in [21], [30] for the purpose of approximation theory. They are simply defined as tensor products of one-dimensional wavelets, allowing different dilations factors in different directions, as opposed to the classical discrete wavelet transform that relies on a single isotropic dilation factor. This key difference enables their use as a tool in the study of anisotropy. Hyperbolic wavelet bases form a nonredundant system by construction, and contain all possible anisotropies. They have been used in 
statistics for the purpose of adaptive estimation of multidimensional curves. Notably, it has been proven in two seminal articles ([37], [38]) that nonlinear thresholding of noisy hyperbolic wavelet coefficients leads to (near) optimal minimax rates of convergence over a wide range of anisotropic smoothness classes; see also the recent work [3] of F. Autin, G. Claeskens and J. M. Freyermuth, where this problem is considered from the maxiset point of view. Other interesting applications of hyperbolic analysis can also be founded in [4] and [5], where the decompositions of fractional Brownian sheets and linear fractional stable sheets are derived and are used to prove sample path properties of these random fields (regularity, Hausdorff dimension of the graph).

A key feature of hyperbolic wavelet bases is that they provide a common dictionary for anisotropic Besov spaces. This result is stated in Theorem 2.6 of Section 2. The critical exponent in anisotropic Besov spaces is related to some $\ell^{p}$ norms of the hyperbolic wavelet coefficients. These mathematical results yield an efficient method for the detection of anisotropy, as detailed in a companion article, where numerical investigations are described; see [42].

We now briefly discuss the pros and cons of both dictionaries. For a fixed anisotropy, Triebel bases display slightly better mathematical properties: an exact characterization of anisotropic Besov spaces, as shown in [49], and a characterization of pointwise smoothness as sharp as in the isotropic case, as shown by H. Ben Braiek and M. Ben Slimane in [7]. One purpose of the present contribution is to show that these two important properties almost hold also for hyperbolic wavelets. In Section 2, "almost characterizations" (i.e., necessary and sufficient conditions that differ by a logarithmic correction) of anisotropic Besov spaces are obtained. Furthermore, if one is not only interested in analysis, but also in simulation, this slight disadvantage (a logarithmic loss, which in applications is irrelevant) is compensated by the advantage of using a basis instead of an overcomplete system. Indeed, generating a random field with prescribed regularity properties requires the use of a basis (using an overcomplete system does not guarantee that the simulated field with coefficients of specific sizes has the expected properties, since nontrivial linear combinations of the building blocks can vanish). To the contrary, with the hyperbolic wavelet basis, one can easily construct toy examples with different multifractal spectra depending on the anisotropy. Our being jointly motivated by analysis and synthesis motivates the choice of a system that permits an interesting trade-off among directional wavelets, in terms of mathematical efficiency and numerical simplicity and robustness, both on the analysis and synthesis sides. The practical relevance of the mathematical tools introduced and studied here are assessed in the companion paper [42].

We now focus on the comparison in terms of pointwise directional smoothness. First, note that this notion has been little studied in the past. To our knowledge, the natural definition which allows for a wavelet characterization was first introduced by M. Ben Slimane in the 90s, see [8], in order to investigate the multifractal properties of anisotropic selfsimilar functions. Partial results when using parabolic bases (i.e., curvelets and Hart Smith transform) have been obtained by J. Sampo and S. Sumetkijakan; see [35], [39] and [43], and references therein. A generaliza- 
tion and its implications in terms of the sizes of coefficients on directional wavelets (the so-called "anisets", which are a mixture of of the wavelet and Gabor transform, where the wavelets can be arbitrarily shrunk in certain directions) were also worked out in [33]. Finally, an "almost" characterization of pointwise directional regularity was recently obtained by $\mathrm{H}$. Ben Braiek and M. Ben Slimane in [7] for the Triebel basis coefficients, where the basis is picked so that its anisotropy parameter is adapted to the type of directional regularity considered. In Section 3, we will obtain a similar result, that, however, relies on the coefficients of the hyperbolic wavelet basis, thus paving the way for the construction of a multifractal formalism. An important difference with [7] is that, here again, a single basis fits all anisotropies. Therefore, as in the case of Besov spaces, the advantage is that nothing needs to be assumed a priori on the particular considered anisotropy. Thus this can be used as a way to detect the specific anisotropy which exists in the data at hand, rather than assuming its particular form beforehand. Note that other decomposition systems have also been used for the detection of local singularities; see for instance [22] and [27], where shearlets and wedgelets are used for the detection of discontinuities along smooth edges.

Now we return to the anisotropic self-similar fields considered in [19] and [42]. Such exactly self-similar models are somewhat toy examples, and, though testing regularity indices on their realizations is an important validation step, their study could prove misleadingly simple (just as, one-dimensional fractional Brownian motion is too simple a model to capture the richness of situations met in real-world data). Natural images are indeed likely to consist of patchworks of different kinds of deformed pieces and therefore can be expected to exhibit more complex scale invariance properties, and only in an approximate way. Multifractal analysis supplies a natural setting for describing such properties, in which different kinds of singularities are mixed. The next step is therefore to combine anisotropy and multifractality. To this end, a new form of multifractal analysis is introduced, based on hyperbolic wavelet coefficients, and relating global and local characterizations of regularity. It allows taking into account both scale invariance properties and local anisotropic features of an image. Thus, it provides a new tool for image classification, seen as a refinement of texture classification based on the usual isotropic multifractal analysis, as proposed for instance in [1] and [34]. Section 3 is devoted to the introduction of this new framework. A new multifractal formalism, referred to as the hyperbolic multifractal formalism is introduced. It permits relating local anisotropic regularity of the analyzed image to global quantities called hyperbolic structure functions as is commonly done in multifractal analysis. Note that an alternative multifractal analysis and multifractal formalism were introduced by H. Ben Braiek and M. Ben Slimane in [6], based on Triebel basis coefficients. In their approach, a particular anisotropy is picked, and the corresponding basis is used. The main difference between both approaches is that the approach proposed here is more flexible and can thus be used when anisotropies of several types are present in data.

In the present article, we explore the possibilities generated by the hyperbolic wavelet transform in order to investigate directional regularity, both in global 
(anisotropic Besov spaces) and local (directional pointwise regularity) forms. The underlying motivation is to develop a multifractal formalism relating these two notions (just as the standard multifractal formalism relates the usual Besov spaces with the notion of pointwise (anisotropic) Hölder smoothness; see [34] and references therein). It also aims at obtaining a numerically stable procedure for extracting the anisotropic features existing in natural images as well as information related to the size (fractional dimensions) of the corresponding geometrical sets.

Our results on the characterization of regularity spaces in terms of hyperbolic wavelets are stated in Section 2. Applications to multifractal analysis are detailed in Section 3. We will show how to recover information on the Hausdorff dimensions of the sets of points where a given directional regularity occurs from knowledge of global directional quantities such as the Besov regularity of the data. Such ideas were initially introduced by Parisi and Frisch in the context of hydrodynamic turbulence [40], and extended to the setting of fixed anisotropy by Ben Braiek and Ben Slimane; see [6]. The novelty here is that using hyperbolic wavelets permits dropping the assumption of unique anisotropy, given a priori, in the data. The next step, which we intend to investigate in the future, is to relax another simplifying assumption and allow for local rotations of the anisotropy axes. Detailed proofs of the results stated in Sections 2 and 3 are provided in Section 4.

\section{Anisotropic global regularity and hyperbolic wavelets}

We focus first on the measure of anisotropic global regularity using a common analyzing dictionary: hyperbolic wavelet bases. Here we start by giving a brief account of the relevant functional spaces. Subsequently, we recall some well-known facts about hyperbolic wavelet analysis (see Section 2.2). The main result of the present section is Theorem 2.6, proven in Section 4.1, which allows the determination of the critical directional Besov indices of data by regressions on log-log plots of quantities based on hyperbolic wavelet coefficients (see Section 2.2 for a precise statement).

\subsection{Anisotropic Besov spaces}

Anisotropic Besov spaces were introduced in a completely different context, for the study of semi-elliptic pseudo-differential operators whose symbols have different degrees of smoothness in different directions; see for example [50]; see also [2], and references therein, for a recent use of such spaces for optimal regularity results for the heat equation.

Anisotropic Besov spaces generalize classical (isotropic) Besov spaces, and many results concerning isotropic spaces have been extended to the anisotropic setting; see [16] and [15] for a complete account of the results used in this section, and [48], [52] and [53] for a detailed overview of anisotropic spaces.

A key property of anisotropic Besov spaces is that they are norm invariant (asymptotically in the limit of small scales) with respect to anisotropic scaling. We start by recalling this notion. Let $\alpha=\left(\alpha_{1}, \alpha_{2}\right)$ denote a fixed pair of parameters, 
with $\alpha_{1}, \alpha_{2}>0$ and $\alpha_{1}+\alpha_{2}=2$. In the remainder, such pairs will be referred to as admissible anisotropies. Such pairs quantify the degree of anisotropy of the space $\left(\alpha_{1}=\alpha_{2}=1\right.$ corresponds to the isotropic case). For any $t \geq 0$ and $\xi=\left(\xi_{1}, \xi_{2}\right) \in \mathbb{R}^{2}$, we define the anisotropic scaling by $t^{\alpha} \xi=\left(t^{\alpha_{1}} \xi_{1}, t^{\alpha_{2}} \xi_{2}\right)$. Note that, in this definition and in the following, the coordinate axes are chosen as the anisotropy directions. This particular choice can of course be modified by the introduction of an additional rotation (as envisaged, for example, in [42]).

Anisotropic Besov spaces may be introduced using an anisotropic LittlewoodPaley analysis. Let $\varphi_{0}^{\alpha} \geq 0$ belong to the Schwartz class $\mathcal{S}\left(\mathbb{R}^{2}\right)$ and be such that

$$
\begin{gathered}
\varphi_{0}^{\alpha}(x)=1 \quad \text { if } \quad \sup _{i=1,2}\left|\xi_{i}\right| \leq 1 \\
\text { and } \varphi_{0}^{\alpha}(x)=0 \quad \text { if } \quad \sup _{i=1,2}\left|2^{-\alpha_{i}} \xi\right| \geq 1 .
\end{gathered}
$$

For $j \in \mathbb{N}$, we define

$$
\varphi_{j}^{\alpha}(x)=\varphi_{0}^{\alpha}\left(2^{-j \alpha} \xi\right)-\varphi_{0}^{\alpha}\left(2^{-(j-1) \alpha} \xi\right) .
$$

Then

$$
\sum_{j=0}^{+\infty} \varphi_{j}^{\alpha} \equiv 1
$$

and $\left(\varphi_{j}^{\alpha}\right)_{j \geq 0}$ is called an anisotropic resolution of unity. It satisfies

$$
\operatorname{supp}\left(\varphi_{0}^{\alpha}\right) \subset R_{1}^{\alpha}, \quad \operatorname{supp}\left(\varphi_{j}^{\alpha}\right) \subset R_{j+1}^{\alpha} \backslash R_{j-1}^{\alpha},
$$

where

$$
R_{j}^{\alpha}=\left\{\xi=\left(\xi_{1}, \xi_{2}\right) \in \mathbb{R}^{2} ; \sup _{i=1,2}\left|\xi_{i}\right| \leq 2^{\alpha_{i} j}\right\} .
$$

For $f \in \mathcal{S}^{\prime}\left(\mathbb{R}^{2}\right)$ let

$$
\Delta_{j}^{\alpha} f=\mathcal{F}^{-1}\left(\varphi_{j}^{\alpha} \widehat{f}\right)
$$

The sequence $\left.\left(\Delta_{j}^{\alpha} f\right)_{j \geq 0}\right)$ is called an anisotropic Littlewood-Paley analysis of $f$. The anisotropic Besov spaces are then defined as follows (see [16] and [15]).

Definition 2.1. The Besov space $B_{p, q,|\log |^{\beta}}^{s, \alpha}\left(\mathbb{R}^{2}\right)$, for $0<p \leq+\infty, 0<q \leq+\infty$, $s, \beta \in \mathbb{R}$, is defined by

$$
B_{p, q,|\log |^{\beta}}^{s, \alpha}\left(\mathbb{R}^{2}\right)=\left\{f \in \mathcal{S}^{\prime}\left(\mathbb{R}^{2}\right) ;\left(\sum_{j \geq 0} j^{-\beta q} 2^{j s q}\left\|\Delta_{j}^{\alpha} f\right\|_{p}^{q}\right)^{1 / q}<+\infty\right\} .
$$

This definition does not depend on the chose resolution of unity $\varphi_{0}^{\alpha}$ and the quantity

$$
\|f\|_{B_{p, q,|\log | \beta}^{s, \alpha}}=\left(\sum_{j \geq 0} j^{-\beta q} 2^{j s q}\left\|\Delta_{j}^{\alpha} f\right\|_{p}^{q}\right)^{1 / q},
$$

is a norm (respectively, quasi-norm) on $B_{p, q}^{s, \alpha}\left(\mathbb{R}^{2}\right)$ for $1 \leq p, q \leq+\infty$ (respectively, $0<\min (p, q)<1)$ and with usual modification if $q=+\infty$. 
Remark 2.2. A homogeneous version $\dot{B}_{p, q}^{s, \alpha}\left(\mathbb{R}^{2}\right)$ of the spaces satisfies the property

$$
\left\|f\left(\lambda^{\alpha_{1}} \cdot \lambda^{\alpha_{2}} \cdot\right)\right\|_{\dot{B}_{p, q}^{s, \alpha}}=\lambda^{s}\|f\|_{\dot{B}_{p, q}^{s, \alpha}}
$$

For $B_{p, q}^{s, \alpha}\left(\mathbb{R}^{2}\right)$, this anisotropic homogeneity of the norm is satisfied asymptotically.

As in the isotropic case, anisotropic Besov spaces encompass a large class of classical anisotropic functional spaces (see [48] for details). For example, when $p=q=2$ and $\left(\alpha_{1}, \alpha_{2}\right) \in \mathbb{Q}^{2}$ is an admissible anisotropy, consider $s>0$ such that $s / \alpha_{1}$ and $s / \alpha_{2}$ are both integers. Then the anisotropic Sobolev space

$$
H^{s, \alpha}\left(\mathbb{R}^{2}\right)=\left\{f \in L^{2}\left(\mathbb{R}^{2}\right) \text { such that } \frac{\partial^{s / \alpha_{1}} f}{\partial x_{1}} \in L^{2}\left(\mathbb{R}^{2}\right) \text { and } \frac{\partial^{s / \alpha_{2}} f}{\partial x_{2}} \in L^{2}\left(\mathbb{R}^{2}\right)\right\},
$$

coincides with the Besov space $B_{2,2}^{s, \alpha}\left(\mathbb{R}^{2}\right)$.

In the special case where $p=q=\infty$, the spaces $B_{\infty, \infty}^{s, \alpha}\left(\mathbb{R}^{2}\right)$ are called anisotropic Hölder spaces and are denoted by $\mathcal{C}_{|\log |^{u}}^{s, \alpha}\left(\mathbb{R}^{2}\right)$. These spaces also admit a finite difference characterization that we recall now (see also [48] for details).

Let $\left(e_{1}, e_{2}\right)$ denote the standard basis of $\mathbb{R}^{2}$. For a function $f: \mathbb{R}^{2} \rightarrow \mathbb{R}$, $\ell \in\{1,2\}$ and $t \in \mathbb{R}$ one defines

$$
\Delta_{t, \ell}^{1} f(x)=f\left(x+t e_{\ell}\right)-f(x) .
$$

The difference of order $M \geq 2$ of a function $f$, in the direction $e_{\ell}$, is defined iteratively by

$$
\Delta_{t, \ell}^{M} f(x)=\Delta_{t, \ell} \Delta_{t, \ell}^{M-1} f(x)
$$

One then has:

Proposition 2.3. Let $\alpha=\left(\alpha_{1}, \alpha_{2}\right) \in\left(\mathbb{R}_{*}^{+}\right)^{2}$ be such that $\alpha_{1}+\alpha_{2}=2$, s $>0$, $u \in \mathbb{R}$, and $f: \mathbb{R}^{2} \rightarrow \mathbb{R}$. The function $f$ belongs to the anisotropic Hölder space $\mathcal{C}_{|\log |^{u}}^{s, \alpha}\left(\mathbb{R}^{2}\right)$ if

$$
\|f\|_{L^{\infty}\left(\mathbb{R}^{2}\right)}+\sum_{\ell=1}^{2} \sup _{t>0} \frac{\left\|\Delta_{t, \ell}^{M_{\ell}} f(x)\right\|_{L^{\infty}\left(\mathbb{R}^{2}\right)}}{|t|^{s / \alpha_{\ell}}|\log (|t|)|^{u}}<+\infty,
$$

where, for any $\ell \in\{1,2\}, M_{\ell}=\left[s / \alpha_{\ell}\right]+1$.

\subsection{Hyperbolic wavelet characterization of anisotropic Besov spaces}

We state our first main result which is a hyperbolic wavelet characterization of anisotropic Besov spaces.

We first recall the definition of the hyperbolic wavelet bases as tensor products of two unidimensional wavelet bases (see [21]) and second state Theorem 2.6, proved later, in Section 4.1.

Definition 2.4. Let $\psi$ denote the unidimensional Meyer wavelet and $\varphi$ the associated scaling function. The hyperbolic wavelet basis is defined as the system $\left\{\psi_{j_{1}, j_{2}, k_{1}, k_{2}},\left(j_{1}, j_{2}\right) \in\left(\mathbb{Z}^{+} \cup\{-1\}\right)^{2},\left(k_{1}, k_{2}\right) \in \mathbb{Z}^{2}\right\}$, where 
- if $j_{1}, j_{2} \geq 0$,

$$
\psi_{j_{1}, j_{2}, k_{1}, k_{2}}\left(x_{1}, x_{2}\right)=\psi\left(2^{j_{1}} x_{1}-k_{1}\right) \psi\left(2^{j_{2}} x_{2}-k_{2}\right) ;
$$

- if $j_{1}=-1$ and $j_{2} \geq 0$

$$
\psi_{-1, j_{2}, k_{1}, k_{2}}\left(x_{1}, x_{2}\right)=\varphi\left(x_{1}-k_{1}\right) \psi\left(2^{j_{2}} x_{2}-k_{2}\right) ;
$$

- if $j_{1} \geq 0$ and $j_{2}=-1$

$$
\psi_{j_{1},-1, k_{1}, k_{2}}\left(x_{1}, x_{2}\right)=\psi\left(2^{j_{1}} x_{1}-k_{1}\right) \varphi\left(x_{2}-k_{2}\right) ;
$$

- if $j_{1}=j_{2}=-1$

$$
\psi_{-1,-1, k_{1}, k_{2}}\left(x_{1}, x_{2}\right)=\varphi\left(x_{1}-k_{1}\right) \varphi\left(x_{2}-k_{2}\right) .
$$

For any $f \in \mathcal{S}^{\prime}\left(\mathbb{R}^{2}\right)$, one defines its hyperbolic wavelet coefficients as follows:

$$
\begin{array}{rlrl}
c_{j_{1}, j_{2}, k_{1}, k_{2}} & =2^{j_{1}+j_{2}}\left\langle f, \psi_{j_{1}, j_{2}, k_{1}, k_{2}}\right\rangle & \text { if } j_{1}, j_{2} \geq 0 \\
c_{j_{1},-1, k_{1}, k_{2}} & =2^{j_{1}}\left\langle f, \psi_{j_{1}, j_{2}, k_{1}, k_{2}}\right\rangle & & \text { if } j_{1} \geq 0 \text { and } j_{2}=-1, \\
c_{-1, j_{2}, k_{1}, k_{2}} & =2^{j_{2}}\left\langle f, \psi_{j_{1}, j_{2}, k_{1}, k_{2}}\right\rangle & & \text { if } j_{1}=-1 \text { and } j_{2} \geq 0, \\
c_{-1,-1, k_{1}, k_{2}} & =\left\langle f, \psi_{j_{1}, j_{2}, k_{1}, k_{2}}\right\rangle & & \text { if } j_{1}=j_{2}=-1 .
\end{array}
$$

Remark 2.5. We have chosen a $L^{1}$-normalization for the wavelet coefficients, as this is best suited to scale invariance.

The main result of this section is a hyperbolic wavelet characterization of the spaces $B_{p, q,|\log |^{\beta}}^{s, \alpha}\left(\mathbb{R}^{2}\right)$, up to a logarithmic correction. In what follow, some notations is needed. For any $\left(j_{1}, j_{2}\right) \in(\mathbb{N} \cup\{-1\})^{2}$, define

$$
\| c_{j_{1}, j_{2}, \cdot, \cdot \|_{\ell^{p}}}=\left(\sum_{\left(k_{1}, k_{2}\right) \in \mathbb{Z}^{2}}\left|c_{j_{1}, j_{2}, k_{1}, k_{2}}\right|^{p}\right)^{1 / p} .
$$

Let $\alpha=\left(\alpha_{1}, \alpha_{2}\right)$ be an admissible anisotropy. We define the following subsets of $(\mathbb{N} \cup\{-1\})^{2}:$

$$
\begin{gathered}
\Gamma_{j}^{(H L)}(\alpha)=\left\{\left[(j-1) \alpha_{1}\right]-1, \ldots,\left[j \alpha_{1}\right]+1\right\} \times\left\{0, \ldots,\left[(j-1) \alpha_{2}\right]-1\right\}, \\
\Gamma_{j}^{(L H)}(\alpha)=\left\{0, \ldots,\left[(j-1) \alpha_{1}\right]-1\right\} \times\left\{\left[(j-1) \alpha_{2}\right]-1, \ldots,\left[j \alpha_{2}\right]+1\right\}, \\
\Gamma_{j}^{(H H)}(\alpha)=\left\{\left[(j-1) \alpha_{1}\right]-1, \ldots,\left[j \alpha_{1}\right]+1\right\} \times\left\{\left[(j-1) \alpha_{2}\right]-1, \ldots,\left[j \alpha_{2}\right]+1\right\}, \\
\text { and } \Gamma_{j}(\alpha)=\Gamma_{j}^{(H L)}(\alpha) \cup \Gamma_{j}^{(L H)}(\alpha) \cup \Gamma_{j}^{(H H)}(\alpha) .
\end{gathered}
$$

Now we state our hyperbolic wavelet characterization of anisotropic Besov spaces. 
Theorem 2.6. Let $\alpha=\left(\alpha_{1}, \alpha_{2}\right)$ be an admissible anisotropy, $(s, \beta) \in \mathbb{R}^{2}$ and let $(p, q) \in(0,+\infty]^{2}$. Let $f \in \mathcal{S}^{\prime}\left(\mathbb{R}^{2}\right)$. If $p \geq 1$ let be $p^{*}=\max \left(p, p^{\prime}\right)$ and let $p_{*}=\min \left(p, p^{\prime}\right)$ with $1 / p+1 / p^{\prime}=1$. Set

$$
r_{1}= \begin{cases}q(1 / p-1)+\max (q-1,0) & \text { if } p \leq 1 \\ \max \left(q / p_{*}-1,0\right), & \text { if } p>1\end{cases}
$$

and

$$
r_{2}= \begin{cases}1, & \text { if } p<1, \\ \max \left(1-q / p^{*}, 0\right) & \text { if } p \geq 1 .\end{cases}
$$

1. Set $\beta=\beta(p, q)=\max (1 / p-1,0)+\max (1-1 / q, 0)$. If

$$
\left(\sum_{j \in \mathbb{N}_{0}} j^{r_{1}-\beta q} 2^{j s q} \sum_{\left(j_{1}, j_{2}\right) \in \Gamma_{j}(\alpha)} 2^{-\left(j_{1}+j_{2}\right) q / p} \| c_{j_{1}, j_{2}, \cdot, \cdot \|_{\ell^{p}}^{q}}\right)^{1 / q}<+\infty,
$$

then $f \in B_{p, q,|\log |^{\beta}}^{s, \alpha}\left(\mathbb{R}^{2}\right)$ (with the usual modifications when $q=\infty$ ).

2. Conversely,

(a) if $q<\infty$ and $f \in B_{p, q,|\log |^{\beta}}^{s, \alpha}\left(\mathbb{R}^{2}\right)$ then

$$
\left(\sum_{j \in \mathbb{N}_{0}} j^{-r_{2}-\beta q-1} 2^{j s q} \sum_{\left(j_{1}, j_{2}\right) \in \Gamma_{j}(\alpha)} 2^{-\left(j_{1}+j_{2}\right) q / p} \| c_{j_{1}, j_{2}, \cdot, \cdot \|_{\ell^{p}}^{q}}\right)^{1 / q}<+\infty ;
$$

(b) if $f \in B_{p, \infty,|\log |^{\beta}}^{s, \alpha}\left(\mathbb{R}^{2}\right)$ then

$$
\max _{j \in \mathbb{N}_{0}} j^{-\beta} 2^{j s} \max _{\left(j_{1}, j_{2}\right) \in \Gamma_{j}(\alpha)} 2^{-\left(j_{1}+j_{2}\right) / p} \| c_{j_{1}, j_{2}, \cdot, \cdot \|_{\ell^{p}}<+\infty}
$$

Note that in the special case $p=q=2$, that is for anisotropic Sobolev spaces, the logarithmic correction disappears.

Theorem 2.7. Let $\alpha$ be an admissible anisotropy, and let $s \in \mathbb{R}$. Let $f \in \mathcal{S}^{\prime}\left(\mathbb{R}^{2}\right)$. The following assertions are equivalent:

(i) $f \in H^{s, \alpha}\left(\mathbb{R}^{2}\right)=B_{2,2}^{s, \alpha}\left(\mathbb{R}^{2}\right)$.

(ii)

$$
\left(\sum_{j \in \mathbb{N}_{0}} 2^{2 j s} \sum_{\left(j_{1}, j_{2}\right) \in \Gamma_{j}(\alpha)} 2^{-\left(j_{1}+j_{2}\right)}\left\|c_{j_{1}, j_{2}, \cdot, \cdot}\right\|_{\ell^{2}}^{2}\right)^{1 / 2}<+\infty .
$$

Theorem 2.6 is proved in Section 4.1.

In particular, for $p=q=\infty$, the following "almost" characterization of anisotropic Hölder spaces by means of hyperbolic wavelets holds:

Proposition 2.8. Let $\alpha=\left(\alpha_{1}, \alpha_{2}\right)$ be an admissible anisotropy, let $(s, \beta) \in \mathbb{R}^{2}$, and let $f \in \mathcal{S}^{\prime}\left(\mathbb{R}^{2}\right)$.

(i) If $f \in \mathcal{C}^{s, \alpha}\left(\mathbb{R}^{2}\right)$ then there exists $C>0$ such that for all $j \in \mathbb{N} \cup\{-1\}$ and any $\left(j_{1}, j_{2}\right) \in \Gamma_{j}(\alpha)$,

$$
\left\|c_{j_{1}, j_{2}, \cdot, \cdot}\right\|_{\ell \infty} \leq C 2^{-j s}
$$


(ii) Conversely, if there exists $C>0$ such that for all $j \in \mathbb{N} \cup\{-1\}$ and any $\left(j_{1}, j_{2}\right) \in \Gamma_{j}(\alpha)$

$$
\left\|c_{j_{1}, j_{2}, \cdot, \cdot}\right\|_{\ell \infty} \leq C \frac{2^{-j s}}{2+j}
$$

then $f \in \mathcal{C}^{s, \alpha}\left(\mathbb{R}^{2}\right)$.

\section{Hyperbolic multifractal analysis}

Now we are interested in the simultaneous analysis of local regularity properties and anisotropic features of a function. To this end, we construct a new method of multifractal analysis, referred to as hyperbolic multifractal analysis. Recall that in the isotropic case, the purpose of multifractal analysis is to provide information on pointwise singularities of functions. Multifractal functions are usually such that their local regularity jumps erratically from point to point, so that it is not possible to estimate the regularity index (defined below) of a function at a given point. Instead, the relevant information consists of the "sizes" of the sets of points where the regularity index takes a given value. This "size" is formalized mathematically as the Hausdorff dimension. The function that associates the dimension of the set of points sharing the same regularity index with this index is referred to as the spectrum of singularities (or multifractal spectrum). The goal of a multifractal formalism is to provide a method for measuring the spectrum of singularities from quantities that can actually be computed from real-world data. We extend this approach to the anisotropic setting. First we recall that, in the case where the anisotropy of the analyzing space is fixed, this has already been achieved. See [7] for anisotropic pointwise regularity analysis using Triebel bases and [6] for the corresponding anisotropic multifractal formalism. Here, we generalize this previous work, developing a multifractal analysis that does not rely on a priori knowledge of regularity and takes into account all possible anisotropies. Note that for a fixed anisotropy, both formalisms coincide. Indeed, they are derived from wavelet characterizations of the same functional spaces. Nevertheless, the formalism based on hyperbolic wavelet allows handling simultaneously all possible anisotropies, thus providing more useful algorithms for analyzing real-world data. In addition, the use of hyperbolic wavelet bases offers the possibility to define and synthesize deterministic and stochastic mathematical objects with prescribed anisotropic behavior.

In Section 3.1, the different concepts related to pointwise regularity are recalled. A hyperbolic wavelet criterion is then devised in Section 3.1.2. Our main result, Theorem 3.4, is stated in Section 3.1.2 and proved in Section 4. Hyperbolic wavelet analysis is defined in Section 3.2.2 and the validity of the proposed multifractal formalism is investigated in Theorem 3.7.

\subsection{Anisotropic pointwise regularity and hyperbolic wavelet analysis}

3.1.1. Definitions. We start by recalling the usual notion of pointwise regularity (see [34] for a complete review). 
Definition 3.1. Let $f$ be in $L_{\mathrm{loc}}^{\infty}\left(\mathbb{R}^{2}\right)$ and let $s>0$. The function $f$ belongs to the space $\mathcal{C}_{|\log |^{\beta}}^{s}\left(x_{0}\right)$ if there exist $C>0, \delta>0$, and $P_{x_{0}}$, a polynomial of degree less than $s$, such that

$$
\text { if }\left|x-x_{0}\right| \leq \delta, \quad\left|f(x)-P_{x_{0}}(x)\right| \leq C\left|x-x_{0}\right|^{s} \cdot\left|\log \left(\left|x-x_{0}\right|\right)\right|^{\beta},
$$

where $|\cdot|$ is the usual Euclidean norm on $\mathbb{R}^{2}$. If $\beta=0$, the space $\mathcal{C}_{|\log |^{0}}^{s}\left(x_{0}\right)$ is denoted simply $\mathcal{C}^{s}\left(x_{0}\right)$.

Anisotropic pointwise regularity is defined as follows. Let $P$ denote a polynomial of the form

$$
P\left(t_{1}, t_{2}\right)=\sum_{\left(\beta_{1}, \beta_{2}\right) \in \mathbb{N}^{2}} a_{\beta_{1}, \beta_{2}} t_{1}^{\beta_{1}} t_{2}^{\beta_{2}},
$$

and let $\alpha=\left(\alpha_{1}, \alpha_{2}\right)$ be an admissible anisotropy. The $\alpha$-homogeneous degree of the polynomial $P$ is defined by

$$
d_{\alpha}(P)=\sup \left\{\alpha_{1} \beta_{1}+\alpha_{2} \beta_{2}, a_{\beta_{1}, \beta_{2}} \neq 0\right\} .
$$

Finally, for any $t=\left(t_{1}, t_{2}\right) \in \mathbb{R}^{2}$, the $\alpha$-homogeneous norm is denoted by

$$
|t|_{\alpha}=\left|t_{1}\right|^{1 / \alpha_{1}}+\left|t_{2}\right|^{1 / \alpha_{2}} .
$$

Now we can define the spaces $\mathcal{C}_{|\log | \beta}^{s, \alpha}\left(x_{0}\right)$.

Definition 3.2. Let $f \in L_{\mathrm{loc}}^{\infty}\left(\mathbb{R}^{2}\right)$, let $\alpha=\left(\alpha_{1}, \alpha_{2}\right)$ be an admissible anisotropy, and let $|\cdot|_{\alpha}, s>0$, and $\beta \in \mathbb{R}$. The function $f$ belongs to $\mathcal{C}_{|\log |^{\beta}}^{s, \alpha}\left(x_{0}\right)$ if there exist $C>0, \delta>0$, and $P_{x_{0}}$ a polynomial with $\alpha$-homogeneous degree less than $s$, such that

$$
\text { if }\left|x-x_{0}\right|_{\alpha} \leq \delta, \quad\left|f(x)-P_{x_{0}}(x)\right| \leq C\left|x-x_{0}\right|_{\alpha}^{s} \cdot\left|\log \left(\left|x-x_{0}\right|_{\alpha}\right)\right|^{\beta} .
$$

If $\beta=0$, the space $\mathcal{C}_{|\log |^{0}}^{s, \alpha}\left(x_{0}\right)$ is denoted simply $\mathcal{C}^{s, \alpha}\left(x_{0}\right)$.

Thus the pointwise anisotropic exponent of a locally bounded function $f$ at $x_{0}$ can be defined by:

$$
h_{f, \alpha}\left(x_{0}\right)=\sup \left\{s, f \in \mathcal{C}^{s, \alpha}\left(x_{0}\right)\right\} .
$$

The link between global and pointwise anisotropic regularity is given by:

Proposition 3.3. If $f \in B_{\infty, \infty}^{s, \alpha}\left(\mathbb{R}^{2}\right)=\mathcal{C}^{s, \alpha}\left(\mathbb{R}^{2}\right)$ then $f$ belongs to $C^{s, \alpha}\left(x_{0}\right)$ for all $x_{0} \in \mathbb{R}^{2}$.

Conversely, if $f$ belongs to $C^{s, \alpha}\left(x_{0}\right)$ for all $x_{0} \in \mathbb{R}^{2}$ with a constant $C$ in (3.1) independent of $x_{0}$, then $f \in B_{\infty, \infty}^{s, \alpha}\left(\mathbb{R}^{2}\right)$.

Proposition 3.3 is a direct consequence of the proof of Proposition 2 of [7], which gives the wavelet characterization of $C^{s, \alpha}\left(x_{0}\right)$ using the Triebel wavelet basis, where it is easy to check that constants appearing in the wavelet characterizations are independent of $x_{0}$ when this is the case for $C$ in (3.1).

The reader is refered to [7] and [33] for more details about the material of this section. 
3.1.2. A hyperbolic wavelet criterion. As in the usual isotropic setting (see [34]), the pointwise anisotropic Hölder regularity of a function is closely related to the rate of decay of its wavelet leaders. The usual definition of wavelet leaders needs to be adapted to the hyperbolic setting.

For any $\left(j_{1}, j_{2}, k_{1}, k_{2}\right)$, let $\lambda\left(j_{1}, j_{2}, k_{1}, k_{2}\right)$ denote the dyadic rectangle

$$
\lambda=\lambda\left(j_{1}, j_{2}, k_{1}, k_{2}\right)=\left[\frac{k_{1}}{2^{j_{1}}}, \frac{k_{1}+1}{2^{j_{1}}}\right) \times\left[\frac{k_{2}}{2^{j_{2}}}, \frac{k_{2}+1}{2^{j_{2}}}\right),
$$

and let $c_{\lambda}$ stand for $c_{j_{1}, j_{2}, k_{1}, k_{2}}$. The hyperbolic wavelet leaders $d_{\lambda}$, associated with $\lambda$, can now be defined by

$$
d_{\lambda}=\sup _{\lambda^{\prime} \subset \lambda}\left|c_{\lambda^{\prime}}\right|
$$

For any $x_{0}=(a, b) \in \mathbb{R}^{2}$, let

$$
3 \lambda_{j_{1}, j_{2}}\left(x_{0}\right)=\left[\frac{\left[2^{j_{1}} a\right]-1}{2^{j_{1}}}, \frac{\left[2^{j_{1}} a\right]+2}{2^{j_{1}}}\right) \times\left[\frac{\left[2^{j_{2}} b\right]-1}{2^{j_{2}}}, \frac{\left[2^{j_{2}} b\right]+2}{2^{j_{2}}}\right)
$$

(where $[\cdot]$ denotes the integer part), and

$$
d_{j_{1}, j_{2}}\left(x_{0}\right)=\sup _{\lambda^{\prime} \subset 3 \lambda_{j_{1}, j_{2}}\left(x_{0}\right)}\left|c_{\lambda^{\prime}}\right| .
$$

The hyperbolic wavelet leader criterion for pointwise regularity can be stated as:

Theorem 3.4. Let $s>0$ and let $\alpha=\left(\alpha_{1}, \alpha_{2}\right) \in\left(\mathbb{R}_{+}^{*}\right)^{2}$ be such that $\alpha_{1}+\alpha_{2}=2$.

1. Assume that $f \in \mathcal{C}^{s, \alpha}\left(x_{0}\right)$. There exists some $C>0$ such that for any $j_{1}, j_{2} \in \mathbb{N} \cup\{-1\}$ one has

$$
\left|d_{j_{1}, j_{2}}\left(x_{0}\right)\right| \leq C 2^{-\max \left(j_{1} / \alpha_{1}, j_{2} / \alpha_{2}\right) s} .
$$

2. Conversely, assume that $f$ is uniformly Hölder, that is there exists some $\varepsilon_{0}^{*}>0$ such that $f \in \mathcal{C}^{\varepsilon_{0}^{*}}\left(\mathbb{R}^{2}\right)$. If (3.4) holds, then $f \in \mathcal{C}_{|\log |^{2}}^{s, \alpha}\left(x_{0}\right)$.

The proofs are given in Section 4 .

\subsection{Anisotropic multifractal analysis}

3.2.1. Hausdorff dimension and spectrum of singularities. The Hausdorff dimension is defined through the Hausdorff measure (see [23] for details). The best covering of a set $E \subset \mathbb{R}^{d}$ by sets of diameters less than $\varepsilon$ can be estimated

$$
\mathcal{H}_{\varepsilon}^{\delta}(E)=\inf \left\{\sum_{i=1}^{\infty}\left|E_{i}\right|^{\delta}, E \subset \bigcup_{i=1}^{\infty} E_{i},\left|E_{i}\right| \leq \varepsilon\right\} .
$$

Clearly, $\mathcal{H}_{\varepsilon}^{\delta}$ is an outer measure. The Hausdorff measure is defined as the (possibly infinite or vanishing) limit $\mathcal{H}_{\epsilon}^{\delta}$ as $\varepsilon$ goes to 0 .

The Hausdorff measure is decreasing as $\delta$ goes to infinity. Moreover, $\mathcal{H}^{\delta}(E)>0$ implies $\mathcal{H}^{\delta^{\prime}}(E)=\infty$ if $\delta^{\prime}<\delta$. Therefore the following definition is meaningful. 
Definition 3.5. The Hausdorff dimension $\operatorname{dim}_{H}(E)$ of a set $E \subset \mathbb{R}^{d}$ is defined by

$$
\operatorname{dim}_{H}(E)=\sup \left\{\delta: \mathcal{H}^{\delta}(E)=\infty\right\} .
$$

With this definition, $\operatorname{dim}_{H}(\emptyset)=-\infty$.

We now define the hyperbolic spectrum of singularities of a locally bounded function using the Hausdorff dimension.

Definition 3.6. Let $f$ be a locally bounded function and let $\alpha$ be an admissible anisotropy. The iso-anisotropic-Hölder set are defined by

$$
E_{f}(H, \alpha)=\left\{x \in \mathbb{R}^{2}, h_{f, \alpha}(x)=H\right\}
$$

where the anisotropic pointwise Hölder $h_{f, \alpha}(x)$ was defined in (3.2).

The hyperbolic spectrum of singularities of $f$ is then defined by

$$
\begin{aligned}
d:\left(\mathbb{R}^{+} \cup\{\infty\}\right) \times(0,2) & \rightarrow \mathbb{R}^{+} \cup\{-\infty\} \\
(H, a) & \mapsto \operatorname{dim}_{H}\left(E_{f}(H,(a, 2-a))\right) .
\end{aligned}
$$

3.2.2. Hyperbolic wavelet leader multifractal formalism. It is not always possible to compute theoretically the spectrum of singularities of a given function. A multifractal formalism thus consists of a practical procedure that yields the convex hull of the function $d$, through the construction of structure functions and the use of the Legendre transform. In the classical case, these formalisms are variants of a derivation proposed by Parisi and Frisch [40]. The hyperbolic wavelet leader multifractal formalism described below is aimed at extending the procedure to where both anisotropy and singularities are studied jointly.

Hyperbolic partition functions of a locally bounded function are defined as

$$
S(j, p, \alpha)=2^{-2 j} \sum_{\left(j_{1}, j_{2}\right) \in \Gamma_{j}(\alpha)} \sum_{\left(k_{1}, k_{2}\right) \in \mathbb{Z}^{2}} d_{j_{1}, j_{2}, k_{1}, k_{2}}^{p},
$$

where $\Gamma_{j}(\alpha)$ is defined in equation (2.2) of Section 2.2.

From the definition of an anisotropic scaling function (or scaling exponents)

$$
\omega_{f}(p, \alpha)=\liminf _{j \rightarrow \infty} \frac{\log S(j, p, \alpha)}{\log 2^{-j}} .
$$

We define the hyperbolic Legendre spectrum by

$$
\mathcal{L}_{f}(H, \alpha)=\inf _{p \in \mathbb{R}^{*}}\left\{H p-\omega_{f}(p,(\alpha, 2-\alpha))+2\right\} .
$$

Qualitatively, the hyperbolic Legendre spectrum and the hyperbolic spectrum of singularities $d_{f}(H, a)$ are expected to coincide, while Theorem 3.7 actually provides an upper bound relationship.

Theorem 3.7. Let $f$ be a uniform Hölder function. Then the inequality

$$
\forall(H, a) \in\left(\mathbb{R}_{+}^{*}\right) \times(0,2), \quad d_{f}(H, a) \leq \mathcal{L}_{f}(H, a)
$$

holds. 
Note that, if equality holds in (3.8), that is, if

$$
\forall(H, a) \in\left(\mathbb{R}_{+}^{*}\right) \times(0,2), \quad d(H, a)=\mathcal{L}_{f}(H, a),
$$

then $f$ is said to satisfy the hyperbolic multifractal formalism.

From an applied perspective, (3.5), (3.6), and (3.7) constitute the core of the practical procedure enabling computation of the hyperbolic Legendre spectrum from the hyperbolic wavelet leaders computed from the data being analyzed.

\section{Proofs}

\subsection{Proof of Theorem 2.6}

4.1.1. Hyperbolic Littlewood-Paley characterization of $B_{p, q}^{s, \alpha}\left(\mathbb{R}^{2}\right)$. Let $\theta_{0} \in \mathcal{S}\left(\mathbb{R}, \mathbb{R}^{+}\right)$be supported on $[-2,2]$ and such that $\theta_{0}=1$ on $[-1,1]$. For any $j \in \mathbb{N}$, define

$$
\theta_{j}=\theta_{0}\left(2^{-j} \cdot\right)-\theta_{0}\left(2^{-(j-1)} \cdot\right)
$$

such that $\sum_{j \geq 0} \theta_{j}(\cdot)=1$ is one-dimensional resolution of the unity.

Observe that, for any $j \geq 1, \operatorname{supp}\left(\theta_{j}\right) \subset\left\{2^{j-1} \leq|\xi| \leq 2^{j+1}\right\}$.

Remark 4.1. In the following, the function $\theta_{0}$ can be chosen with an arbitrary compact support. This does not change the main results even if technical details of proofs and lemmas have to be adapted. This means that the Fourier transform of a Meyer scaling function for $\theta_{0}$ can be chosen as one likes.

Definition 4.2. 1) For any $j, \ell \geq 0$, and any $\xi=\left(\xi_{1}, \xi_{2}\right) \in \mathbb{R}^{2}$ set

$$
\phi_{j_{1}, j_{2}}\left(\xi_{1}, \xi_{2}\right)=\theta_{j_{1}}\left(\xi_{1}\right) \theta_{j_{2}}\left(\xi_{2}\right) \text {. }
$$

For any $j_{1}, j_{2} \geq 0, \phi_{j_{1}, j_{2}}$ belongs to $\mathcal{S}\left(\mathbb{R}^{2}\right)$ and is compactly supported in

$$
\left\{2^{j_{1}} \leq\left|\xi_{1}\right| \leq 2^{j_{1}+1}\right\} \times\left\{2^{j_{2}} \leq\left|\xi_{2}\right| \leq 2^{j_{2}+1}\right] .
$$

Further $\sum_{j_{1} \geq 0} \sum_{j_{2} \geq 0} \phi_{j_{1}, j_{2}}=1$ and $\left(\phi_{j_{1}, j_{2}}\right)_{\left(j_{1}, j_{2}\right) \in \mathbb{N}^{2}}$ is called a hyperbolic resolution of unity.

2) For $f \in \mathcal{S}^{\prime}\left(\mathbb{R}^{2}\right)$ and $j_{1}, j_{2} \geq 0$ set

$$
\Delta_{j_{1}, j_{2}} f=\mathcal{F}^{-1}\left(\phi_{j_{1}, j_{2}} \widehat{f}\right) .
$$

The sequence $\left(\left(\Delta_{j_{1}, j_{2}} f\right)_{j_{1}, j_{2} \geq 0}\right)$ is called a hyperbolic Littlewood-Paley analysis of $f$.

In the remainder of the section, we are given $\alpha=\left(\alpha_{1}, \alpha_{2}\right)$, a fixed admissible anisotropy, and $\left(\varphi_{j}^{\alpha}\right)_{j \geq 0}$, an anisotropic resolution of unity. Then, for any $j \geq 0$ one defines the functions

$$
g_{j}^{\alpha}=\sum_{j_{1}, j_{2} \in \Gamma_{j}(\alpha)} \phi_{j_{1}, j_{2}},
$$

where the sets $\Gamma_{j}(\alpha)$ are defined in $(2.2)$. 
Remark 4.3. Hyperbolic Littlewood-Paley analysis is used in the definition of spaces of mixed smoothness. We refer to [44] for a study of these spaces and to [45] for their link with tensor products of Besov spaces and their hyperbolic wavelet characterizations.

We now give the following hyperbolic Littlewood-Paley characterization of anisotropic Besov spaces:

Theorem 4.4. Let $s \in \mathbb{R}$ and $(p, q) \in(0,+\infty]^{2}$. If $p \geq 1$ let $p^{*}=\max \left(p, p^{\prime}\right)$ and $p_{*}=\min \left(p, p^{\prime}\right)$, with $1 / p+1 / p^{\prime}=1$.

1. (a) If $q<\infty$ and

$$
\begin{gathered}
\left(\sum_{j \geq 0} j^{r_{1}} \cdot j^{-\beta q} 2^{j s q} \sum_{\left(j_{1}, j_{2}\right) \in \Gamma_{j}(\alpha)}\left\|\Delta_{j_{1}, j_{2}}(f)\right\|_{p}^{q}\right)^{1 / q}<+\infty, \\
\text { with } r_{1}= \begin{cases}q(1 / p-1)+\max (q-1,0) & \text { if } p \leq 1, \\
\max \left(q / p_{*}-1,0\right), & \text { if } p>1,\end{cases}
\end{gathered}
$$

then $f \in B_{p, q,|\log |^{\beta}}^{s, \alpha}\left(\mathbb{R}^{2}\right)$.

(b) If

(4.3) $\max _{j \geq 0}\left(j^{\max (1 / p-1,0)+1} \cdot j^{-\beta} 2^{j s} \max _{\left(j_{1}, j_{2}\right) \in \Gamma_{j}(\alpha)}\left\|\Delta_{j_{1}, j_{2}}(f)\right\|_{p}\right)<+\infty$,

then $f \in B_{p, \infty,|\log |^{\beta}}^{s, \alpha}\left(\mathbb{R}^{2}\right)$.

2. (a) If $q<\infty$ and $f \in B_{p, q,|\log |^{\beta}}^{s, \alpha}\left(\mathbb{R}^{2}\right)$ then

$$
\begin{gathered}
\left(\sum_{j \geq 0} j^{-r_{2}} \cdot j^{-\beta q} 2^{j s q} \sum_{\left(j_{1}, j_{2}\right) \in \Gamma_{j}(\alpha)}\left\|\Delta_{j_{1}, j_{2}}(f)\right\|_{p}^{q}\right)^{1 / q}<+\infty, \\
\text { with } r_{2}= \begin{cases}1, & \text { if } p<1, \\
\max \left(1-q / p^{*}, 0\right) & \text { if } p \geq 1 .\end{cases}
\end{gathered}
$$

(b) If $f \in B_{p, \infty,|\log |^{\beta}}^{s, \alpha}\left(\mathbb{R}^{2}\right)$ then

$$
\max _{j \geq 0}\left(j^{-\beta} 2^{j s} \max _{\left(j_{1}, j_{2}\right) \in \Gamma_{j}(\alpha)}\left\|\Delta_{j_{1}, j_{2}}(f)\right\|_{p}\right)<+\infty .
$$

Note, in particular, that for $p=q=2$ the logarithmic correction disappears and Theorem 4.4 gives an exact characterization of anisotropic Sobolev spaces in term of hyperbolic Littlewood-Paley analysis.

The proof of Theorem 4.4 consists of several steps, beginning with:

Lemma 4.5. 1. For any $j \geq 0$ and any $\left(j_{1}, j_{2}\right) \notin \Gamma_{j}(\alpha)$, one has

$$
\operatorname{supp}\left(\varphi_{j}^{\alpha}\right) \cap \operatorname{supp}\left(\phi_{j_{1}, j_{2}}\right)=\emptyset .
$$


2. For any $j \geq 0$ and any $\ell \notin\{j-1, j, j+1\}$, one has

$$
\operatorname{supp}\left(g_{j}^{\alpha}\right) \cap \operatorname{supp}\left(\varphi_{\ell}^{\alpha}\right)=\emptyset .
$$

Proof. Both claims are checked easily since the support of both functions are known (see equation (2.1) and Definition 4.2).

From Lemma 4.5 an intermediate hyperbolic Littlewood-Paley characterization of anisotropic Besov spaces is obtained.

Proposition 4.6. Let $(p, q) \in(0,+\infty]^{2}, s, \beta \in \mathbb{R}$. The following assertions are equivalent:

1. $f \in B_{p, q,|\log |^{\beta}}^{s, \alpha}\left(\mathbb{R}^{2}\right)$.

2 .

$$
\left(\sum_{j \geq 0} j^{-\beta q} 2^{j s q}\left\|\sum_{\left(j_{1}, j_{2}\right) \in \Gamma_{j}(\alpha)}\left[\Delta_{j_{1}, j_{2}}(f)\right]\right\|_{p}^{q}\right)^{1 / q}<+\infty
$$

Proof of Proposition 4.6.

We prove that the inequality (4.6) of Proposition 4.6 implies $f \in B_{p, q,|\log |^{\beta}}^{s, \alpha}\left(\mathbb{R}^{2}\right)$. To this end, point 1 of Lemma 4.5 is used to deduce that, for any $j$,

$$
\varphi_{j}^{\alpha} \widehat{f}=\varphi_{j}^{\alpha}\left(\sum_{\left(j_{1}, j_{2}\right) \in \mathbb{N}^{2}} \phi_{j_{1}, j_{2}}\right) \widehat{f}=\varphi_{j}^{\alpha}\left(\sum_{\left(j_{1}, j_{2}\right) \in \Gamma_{j}(\alpha)} \phi_{j_{1}, j_{2}}\right) \widehat{f}=\varphi_{j}^{\alpha}\left(g_{j}^{\alpha} \widehat{f}\right),
$$

where $g_{j}^{\alpha}$ is defined by (4.1). Observe now that replacing the usual dilation with an anisotropic one gives an anisotropic version of equation (13) in Section 1.5.2 in [50]. More precisely assume that we are given $p \in(0,+\infty], b>0$ and $M \in \mathcal{S}\left(\mathbb{R}^{2}\right)$. There exists $C>0$ not depending on $b$ or $M$ such that for any $h \in L^{p}\left(\mathbb{R}^{2}\right)$ such that $\operatorname{supp}(\widehat{h}) \subset\left\{\xi \in \mathbb{R}^{2}, \sup _{i}\left|\xi_{i}\right| \leq b^{\alpha_{i}}\right\}$ one has

$$
\left\|\mathcal{F}^{-1}(M \mathcal{F} h)\right\|_{L^{p}\left(\mathbb{R}^{2}\right)} \leq C\left\|M\left(b^{\alpha} \cdot\right)\right\|_{H_{2}^{s}\left(\mathbb{R}^{2}\right)}\|h\|_{L^{p}\left(\mathbb{R}^{2}\right)}
$$

where $H_{2}^{s}$ is the usual Bessel potential space and $s>2(1 / \min (p, 1)-1 / 2)$.

Set now $b=2^{j}, M=\varphi_{j}^{\alpha}$ and $\widehat{h}=g_{j}^{\alpha} \widehat{f}$. Since $\varphi_{j}^{\alpha}\left(2^{j \alpha} \cdot\right)=\varphi_{1}^{\alpha}$, there exists some $C>0$ not depending on $j$ such that for any $p \in(0,+\infty]$ and any $f \in L^{p}\left(\mathbb{R}^{2}\right)$

$$
\left\|\Delta_{j}^{\alpha} f\right\|_{L^{p}} \leq C\left\|\sum_{\left(j_{1}, j_{2}\right) \in \Gamma_{j}(\alpha)} \Delta_{j_{1}, j_{2}} f\right\|_{L^{p}}=C\left\|\left(\mathcal{F}^{-1} g_{j}^{\alpha}\right) * f\right\|_{L^{p}}
$$

Then

$$
\|f\|_{B_{p, q,|\log |^{\beta}}^{s, \alpha}}=\left(\sum_{j \geq 0} j^{-\beta q} 2^{j s q}\left\|\Delta_{j}^{\alpha} f\right\|_{L^{p}}^{q}\right)^{1 / q} \leq C\left(\sum_{j \geq 0} j^{-\beta q} 2^{j s q}\left\|\left(\mathcal{F}^{-1} g_{j}^{\alpha}\right) * f\right\|_{L^{p}}^{q}\right)^{1 / q}
$$

which shows point 1 of Proposition 4.6. 
Now we prove the converse assertion. Assume that $f$ belongs to $B_{p, q,|\log |^{\beta}}^{s, \alpha}\left(\mathbb{R}^{2}\right)$. For any $j \geq 0$, point 2 of Lemma 4.5 gives

$$
g_{j}^{\alpha} \widehat{f}=g_{j}^{\alpha}\left(\varphi_{j-1}^{\alpha}+\varphi_{j}^{\alpha}+\varphi_{j+1}^{\alpha}\right) \widehat{f} .
$$

Hence, inequality (4.7) applied with $b=2^{j}, M=g_{j}^{\alpha}$, and $\widehat{h}=\left(\varphi_{j-1}^{\alpha}+\varphi_{j}^{\alpha}+\varphi_{j+1}^{\alpha}\right) \widehat{f}$, gives the existence of a $C>0$ not depending on $j$ or $f$ such that

$$
\left\|\left(\mathcal{F}^{-1} g_{j}^{\alpha}\right) * f\right\|_{L^{p}} \leq C\left\|g_{j}^{\alpha}\left(2^{j \alpha} \cdot\right)\right\|_{H_{2}^{s}}\left\|\left(\mathcal{F}^{-1} \varphi_{j-1}^{\alpha}+\mathcal{F}^{-1} \varphi_{j}^{\alpha}+\mathcal{F}^{-1} \varphi_{j+1}^{\alpha}\right) * f\right\|_{L^{p}}
$$

for any $p \in(0,+\infty]$. Since $\|\cdot\|_{L^{p}}$ is either a norm or a quasi-norm (according to the value of $p$ ), there exists $C>0$ such that

$$
\left\|\left(\mathcal{F}^{-1} g_{j}^{\alpha}\right) * f\right\|_{L^{p}} \leq C\left\|g_{j}^{\alpha}\left(2^{j \alpha} \cdot\right)\right\|_{H_{2}^{s}} \sum_{l=j-1}^{j+1}\left\|\left(\mathcal{F}^{-1} \varphi_{l}^{\alpha}\right) * f\right\|_{L^{p}} .
$$

First we bound $\left\|g_{j}^{\alpha}\left(2^{j \alpha} \cdot\right)\right\|_{H_{2}^{s}}$. To this end, observe that

$$
\begin{aligned}
\mathcal{F}\left[g_{j}^{\alpha}\left(2^{j \alpha} \cdot\right)\right](\xi) & =2^{-j\left(\alpha_{1}+\alpha_{2}\right)} \widehat{g}_{j}\left(2^{-j \alpha} \xi\right)=2^{-2 j} \widehat{g}_{j}\left(2^{-j \alpha} \xi\right) \\
& =\sum_{\left(j_{1}, j_{2}\right) \in \Gamma_{j}(\alpha)} 2^{j_{1}+j_{2}-2 j} \widehat{\theta}_{1}\left(2^{j_{1}-j \alpha_{1}} \xi_{1}\right) \widehat{\theta}_{1}\left(2^{j_{2}-\alpha_{2} j} \xi_{2}\right) .
\end{aligned}
$$

Hence

$$
\begin{aligned}
& \left\|g_{j}^{\alpha}\left(2^{j \alpha} \cdot\right)\right\|_{H_{2}^{s}}^{2} \\
& \quad=\int_{\mathbb{R}^{2}}\left(1+|\xi|^{2}\right)^{s}\left[\sum_{\left(j_{1}, j_{2}\right) \in \Gamma_{j}(\alpha)} 2^{j_{1}+j_{2}-2 j} \widehat{\theta}_{1}\left(2^{j_{1}-j \alpha_{1}} \xi_{1}\right) \widehat{\theta}_{1}\left(2^{j_{2}-\alpha_{2} j} \xi_{2}\right)\right]^{2} \mathrm{~d} \xi \\
& \quad \leq \int_{\mathbb{R}^{2}}\left(1+|\xi|^{2}\right)^{s}\left[\sum_{\left(j_{1}, j_{2}\right) \in \Gamma_{j}(\alpha)} 2^{j_{1}+j_{2}-2 j}\left|\widehat{\theta}_{1}\left(2^{j_{1}-j \alpha_{1}} \xi_{1}\right)\right| \mid \widehat{\theta}_{1}\left(2^{j_{2}-\alpha_{2} j} \xi_{2}\right)\right]^{2} \mathrm{~d} \xi .
\end{aligned}
$$

Since $\theta_{1} \in \mathcal{S}(\mathbb{R})$, for any $M>1$ there exists $C>0$ such that

$$
\left|\widehat{\theta}_{1}(\zeta)\right| \leq \frac{C_{M}}{(1+|\zeta|)^{2 M}} .
$$

Finally

$$
\begin{aligned}
& \left\|g_{j}^{\alpha}\left(2^{j \alpha} \cdot\right)\right\|_{H_{2}^{s}}^{2} \\
& \quad \leq C_{M} \int_{\mathbb{R}^{2}}\left(1+|\xi|^{2}\right)^{s}\left[\sum_{\left(j_{1}, j_{2}\right) \in \Gamma_{j}(\alpha)} \frac{2^{j_{1}+j_{2}-2 j}}{\left(1+\left|2^{j_{1}-j \alpha_{1}} \xi_{1}\right|\right)^{2 M} \cdot\left(1+\left|2^{j_{2}-j \alpha_{2}} \xi_{2}\right|\right)^{2 M}}\right]^{2} \mathrm{~d} \xi \\
& \quad \leq C_{M} \int_{\mathbb{R}^{2}}\left(1+|\xi|^{2}\right)^{s}\left[\sum_{\left(j_{1}, j_{2}\right) \in \Gamma_{j}(\alpha)} \frac{1}{\left(2^{j \alpha_{1}-j_{1}}+\left|\xi_{1}\right|\right)^{2 M} \cdot\left(2^{j \alpha_{2}-j_{2}}+\left|\xi_{2}\right|\right)^{2 M}}\right]^{2} \mathrm{~d} \xi .
\end{aligned}
$$


By the inequality

$$
(a+b)^{2} \geq a \max (b, 1),
$$

valid for any $a>1$ and $b>0$, and applied first with $a=2^{j \alpha_{1}-j_{1}}$ and $b=\left|\xi_{1}\right|$ and then with $a=2^{j \alpha_{2}-j_{2}}$ and $b=\left|\xi_{2}\right|$, there results

$$
\left\|g_{j}^{\alpha}\left(2^{j \alpha} \cdot\right)\right\|_{H_{2}^{s}}^{2} \leq C_{M} \int_{\mathbb{R}^{2}}\left(1+|\xi|^{2}\right)^{s}\left[\sum_{\left(j_{1}, j_{2}\right) \in \Gamma_{j}(\alpha)} \frac{2^{\left(j_{1}-j \alpha_{1}\right) M} 2^{\left(j_{2}-j \alpha_{2}\right) M}}{\max \left(1,\left|\xi_{1}\right|\right)^{M} \max \left(1,\left|\xi_{2}\right|\right)^{M}}\right]^{2} \mathrm{~d} \xi .
$$

With a sufficiently large $M$ it follows that

$$
\sup _{j}\left(\left\|g_{j}^{\alpha}\left(2^{j \alpha} \cdot\right)\right\|_{H_{2}^{s}}\right)<+\infty .
$$

Returning to finding an upper bound of $\left\|\left(\mathcal{F}^{-1} g_{j}^{\alpha}\right) * f\right\|_{L^{p}}$, there exists $C>0$ such that

$$
\left\|\left(\mathcal{F}^{-1} g_{j}^{\alpha}\right) * f\right\|_{L^{p}} \leq C j \sum_{l=j-1}^{j+1}\left\|\left(\mathcal{F}^{-1} \varphi_{l}^{\alpha}\right) * f\right\|_{L^{p}}
$$

and

$$
\sum_{j \geq 0} j^{-\beta q} 2^{j s q}\left\|\left(\mathcal{F}^{-1} g_{j}^{\alpha}\right) * f\right\|_{L^{p}}^{q} \leq C\|f\|_{B_{p, q,|\log | \beta}^{s, \alpha}}^{q}=\sum_{j \geq 0} j^{-\beta q} 2^{j s q}\left\|\Delta_{j}^{\alpha} f\right\|_{L^{p}}^{q}
$$

The last equality shows that if (4.6) holds then $f \in B_{p, q,|\log |^{\beta}}^{s, \alpha}\left(\mathbb{R}^{2}\right)$.

Note that the proof can be shortened in the case $p \geq 1$ using Young's inequality

$$
\left\|\mathcal{F}^{-1}(M \mathcal{F} h)\right\|_{L^{p}\left(\mathbb{R}^{2}\right)} \leq C\left\|\mathcal{F}^{-1} M\right\|_{L^{1}\left(\mathbb{R}^{2}\right)}\|h\|_{L^{p}\left(\mathbb{R}^{2}\right)}
$$

in place of inequality (4.7).

Proof of Theorem 4.4. First we recall that:

- For any $q \in(0,+\infty), n \in \mathbb{N}$, and $\left(a_{1}, \ldots, a_{n}\right) \in\left(\mathbb{R}_{+}\right)^{n}$,

$$
\left(a_{1}+\cdots+a_{n}\right)^{q} \leq n^{\max (q-1,0)}\left(a_{1}^{q}+\cdots+a_{n}^{q}\right) .
$$

- For any $p \in(0,1], n \in \mathbb{N}$, and $\left(f_{1}, \ldots, f_{n}\right) \in L^{p}\left(\mathbb{R}^{2}\right)^{n}$,

$$
\left\|f_{1}+\cdots+f_{n}\right\|_{L^{p}} \leq n^{1 / p-1}\left(\left\|f_{1}\right\|+\cdots+\left\|f_{n}\right\|\right) .
$$

Moreover Lemma 7.1 of [47] applied to the hyperbolic Littlewood-Paley analysis provides the following inequality as a consequence of interpolation of the Plancherel and Minkowski inequalities:

$$
\left[\sum_{j_{1}, j_{2} \in J}\left\|\Delta_{j_{1}, j_{2}} f\right\|_{p}^{p^{*}}\right]^{1 / p^{*}} \leq\left\|\sum_{j_{1}, j_{2} \in J} \Delta_{j_{1}, j_{2}} f\right\|_{p} \leq\left[\sum_{j_{1}, j_{2} \in J}\left\|\Delta_{j_{1}, j_{2}} f\right\|_{p}^{p_{*}}\right]^{1 / p_{*}},
$$

for $p \geq 1$ and any subset $J$ of $\mathbb{N}^{2}$. (Recall that $p_{*}=\min \left(p, p^{\prime}\right)$ and $p^{*}=\max \left(p, p^{\prime}\right)$.) 
Now we prove the first claim of the theorem in the case where $q \neq \infty$. For this, we assume that (4.2) holds and let prove that $f \in B_{p, q,|\log |^{\beta}}^{s, \alpha}\left(\mathbb{R}^{2}\right)$. For $p<1$, by inequalities (4.9) and (4.10) and the fact that $\operatorname{Card}\left(\Gamma_{j}(\alpha)\right) \leq C j$ there exists $C>0$ such that

$$
\left\|\sum_{\left(j_{1}, j_{2}\right) \in \Gamma_{j}(\alpha)} \Delta_{j_{1}, j_{2}} f\right\|_{L^{p}}^{q} \leq C j^{q(1 / p-1)+\max (q-1,0)} \sum_{\left(j_{1}, j_{2}\right) \in \Gamma_{j}(\alpha)}\left\|\Delta_{j_{1}, j_{2}} f\right\|_{L^{p}}^{q} .
$$

Hence,

$$
\begin{aligned}
& \left(\sum_{j \geq 0} j^{-\beta q} 2^{j s q}\left\|\sum_{\left(j_{1}, j_{2}\right) \in \Gamma_{j}(\alpha)} \Delta_{j_{1}, j_{2}} f\right\|_{L^{p}}^{q}\right)^{1 / q} \\
& \leq C\left(\sum_{j \geq 0} j^{q(1 / p-1)+\max (q-1,0)} \cdot j^{-\beta q} 2^{j s q} \sum_{\left(j_{1}, j_{2}\right) \in \Gamma_{j}(\alpha)}\left\|\Delta_{j_{1}, j_{2}} f\right\|_{L^{p}}^{q}\right)^{1 / q} .
\end{aligned}
$$

This proves that, if (4.2) holds, one has

$$
\left(\sum_{j \geq 0} j^{-\beta q} 2^{j s q}\left\|\sum_{\left(j_{1}, j_{2}\right) \in \Gamma_{j}(\alpha)} \Delta_{j_{1}, j_{2}} f\right\|_{L^{p}}^{q}\right)^{1 / q}<\infty .
$$

Finally, by (1) of Proposition 4.6, there results that $f \in B_{p, q,|\log |^{\beta}}^{s, \alpha}\left(\mathbb{R}^{2}\right)$.

If $p \geq 1$, inequality (4.11) with (4.9) gives

$$
\begin{aligned}
\left\|\sum_{\left(j_{1}, j_{2}\right) \in \Gamma_{j}(\alpha)} \Delta_{j_{1}, j_{2}} f\right\|_{L^{p}}^{q} & \leq\left(\sum_{\left(j_{1}, j_{2}\right) \in \Gamma_{j}(\alpha)}\left\|\Delta_{j_{1}, j_{2}} f\right\|_{p}^{p_{*}}\right)^{q / p_{*}} \\
& \leq C j^{\max \left(q / p_{*}-1,0\right)} \sum_{\left(j_{1}, j_{2}\right) \in \Gamma_{j}(\alpha)}\left\|\Delta_{j_{1}, j_{2}} f\right\|_{p}^{q}
\end{aligned}
$$

which also leads to the desired result.

We now treat the case $q=\infty$. In this case, we have

$$
\begin{aligned}
\max _{j \geq 0} j^{-\beta} 2^{j s} \| & \sum_{\left(j_{1}, j_{2}\right) \in \Gamma_{j}(\alpha)} \Delta_{j_{1}, j_{2}} f \|_{L^{p}} \\
& \leq C \max _{j \geq 0} j^{\max \left(\frac{1}{p}-1,0\right)-\beta} 2^{j s} \sum_{\left(j_{1}, j_{2}\right) \in \Gamma_{j}(\alpha)}\left\|\Delta_{j_{1}, j_{2}} f\right\|_{L^{p}} .
\end{aligned}
$$

Hence if (4.6) holds, $f \in B_{p, \infty,|\log |^{\beta}}^{s, \alpha}\left(\mathbb{R}^{2}\right)$.

To prove the converse assertion, assume $f \in B_{p, q,|\log |^{\beta}}^{s, \alpha}\left(\mathbb{R}^{2}\right)$. We first treat the case $p<1$. Observe that for any $j \geq 0$ and any $\left(j_{1}, j_{2}\right) \in \Gamma_{j}(\alpha)$, one has

$$
\phi_{j_{1}, j_{2}} \widehat{f}=\phi_{j_{1}, j_{2}}\left(g_{j-1}^{\alpha}+g_{j}^{\alpha}+g_{j+1}^{\alpha}\right) \widehat{f} .
$$

Note that $\phi_{j_{1}, j_{2}}\left(2^{j \alpha} \cdot\right)$ is bounded in $H_{2}^{s}\left(\mathbb{R}^{2}\right)$ independently of $\left(j_{1}, j_{2}\right) \in \Gamma_{j}(\alpha)$. Hence, by (4.7), there exists $C>0$ not depending on $j$ or $f$ such that, for 
any $\left(j_{1}, j_{2}\right) \in \Gamma_{j}(\alpha)$,

$$
\left\|\left(\mathcal{F}^{-1} \phi_{j_{1}, j_{2}}\right) * f\right\|_{L^{p}} \leq C \sum_{l=j-1}^{j+1}\left\|\left(\mathcal{F}^{-1} g_{l}^{\alpha}\right) * f\right\|_{L^{p}}
$$

Again, two cases have to be distinguished according to whether $q \neq \infty$ or $q=\infty$.

Consider the case $q<\infty$. Observing that $\operatorname{Card}\left(\Gamma_{j}(\alpha)\right) \leq C j$, we deduce that

$$
\sum_{\left(j_{1}, j_{2}\right) \in \Gamma_{j}(\alpha)}\left\|\left(\mathcal{F}^{-1} \phi_{j_{1}, j_{2}}\right) * f\right\|_{L^{p}}^{q} \leq C j \sum_{l=j-1}^{j+1}\left\|\left(\mathcal{F}^{-1} g_{l}^{\alpha}\right) * f\right\|_{L^{p}}^{q},
$$

so

$$
\begin{aligned}
& \sum_{j} j^{-1} j^{-\beta q} 2^{j s q} \sum_{\left(j_{1}, j_{2}\right) \in \Gamma_{j}(\alpha)}\left\|\left(\mathcal{F}^{-1} \phi_{j_{1}, j_{2}}\right) * f\right\|_{L^{p}}^{q} \\
& \leq \sum_{j} j \cdot j^{-1} j^{-\beta q} 2^{j s q}\left\|\left(\mathcal{F}^{-1} g_{j}^{\alpha}\right) * f\right\|_{L^{p}}^{q} .
\end{aligned}
$$

Since in addition the function $f$ is assumed to belong to $B_{p, q,|\log |^{\beta}}^{s, \alpha}\left(\mathbb{R}^{2}\right)$, one has

$$
\sum_{j} j^{-\beta q} 2^{j s q}\left\|\left(\mathcal{F}^{-1} g_{j}^{\alpha}\right) * f\right\|_{L^{p}}^{q}=\sum_{j} j \cdot j^{-1} j^{-\beta q} 2^{j s q}\left\|\left(\mathcal{F}^{-1} g_{j}^{\alpha}\right) * f\right\|_{L^{p}}^{q}<\infty,
$$

which directly yields the required inequality using (4.12).

The case $p \geq 1$ is simpler. Again, inequalities (4.11) and (4.9) give

$$
\begin{aligned}
\left\|\sum_{\left(j_{1}, j_{2}\right) \in \Gamma_{j}(\alpha)} \Delta_{j_{1}, j_{2}} f\right\|_{L^{p}}^{q} & \geq\left(\sum_{\left(j_{1}, j_{2}\right) \in \Gamma_{j}(\alpha)}\left\|\Delta_{j_{1}, j_{2}} f\right\|_{p}^{p^{*}}\right)^{q / p^{*}} \\
& \geq C j^{-\max \left(1-q / p^{*}, 0\right)} \sum_{\left(j_{1}, j_{2}\right) \in \Gamma_{j}(\alpha)}\left\|\Delta_{j_{1}, j_{2}} f\right\|_{p}^{q}
\end{aligned}
$$

which allow us to obtain the desired conclusion.

In the case $q=\infty$, we have

$$
\max _{\left(j_{1}, j_{2}\right) \in \Gamma_{j}(\alpha)}\left\|\left(\mathcal{F}^{-1} \phi_{j_{1}, j_{2}}\right) * f\right\|_{L^{p}}^{q} \leq C \max _{\ell=j-1, j, j+1}\left\|\left(\mathcal{F}^{-1} g_{\ell}^{\alpha}\right) * f\right\|_{L^{p}},
$$

which leads, for a $C>0$, to

$$
\max _{j \geq 0}\left(j^{-\beta} 2^{j s} \max _{\left(j_{1}, j_{2}\right) \in \Gamma_{j}(\alpha)}\left\|\left(\mathcal{F}^{-1} \phi_{j_{1}, j_{2}}\right) * f\right\|_{L^{p}}\right) \leq I,
$$

where

$$
I=C \max _{j \geq 0}\left(j^{-\beta} 2^{j s}\left\|\left(\mathcal{F}^{-1} g_{j}^{\alpha}\right) * f\right\|_{L^{p}}\right) .
$$

That is,

$$
\max _{j_{1}, j_{2} \geq 0}\left(\max \left(\frac{j_{1}}{\alpha_{1}}, \frac{j_{2}}{\alpha_{2}}\right)\right)^{-\beta} 2^{\max \left(j_{1} / \alpha_{1}, j_{2} / \alpha_{2}\right) s}\left\|\left(\mathcal{F}^{-1} \phi_{j_{1}, j_{2}}\right) * f\right\|_{L^{p}} \leq I .
$$


Since, in addition, $f$ is assumed to belong to $B_{p, \infty,|\log |^{\beta}}^{s, \alpha}\left(\mathbb{R}^{2}\right)$, there results

$$
\max j^{-\beta} 2^{j s}\left\|\left(\mathcal{F}^{-1} g_{j}^{\alpha}\right) * f\right\|_{L^{p}}<\infty .
$$

Finally, the required conclusion is obtained by an approach similar to the one used for the previous case.

\subsubsection{Proof of the hyperbolic wavelet characterization of anisotropic}

Besov spaces. First we consider the general case where $(p, q) \in(0,+\infty]^{2}, \beta, s \in \mathbb{R}$ and $\alpha=\left(\alpha_{1}, \alpha_{2}\right)$ is a fixed anisotropy. The intermediate spaces $\mathcal{E}_{p, q,|\log |^{\beta}}^{s, \alpha}\left(\mathbb{R}^{2}\right)$ are defined as the collection of functions $f$ in $\mathcal{S}^{\prime}\left(\mathbb{R}^{2}\right)$ such that

$$
\sum_{j \geq 0} j^{-\beta q} 2^{j s q} \sum_{\left(j_{1}, j_{2}\right) \in \Gamma_{j}(\alpha)}\left\|\Delta_{j_{1}, j_{2}} f\right\|_{p}^{q}<+\infty .
$$

A norm on $\mathcal{E}_{p, q,|\log |^{\beta}}^{s, \alpha}\left(\mathbb{R}^{2}\right)$ is defined by

$$
\|f\|_{\mathcal{E}_{p, q,|\log |^{\beta}}^{s, \alpha}}=\left(\sum_{j \geq 0} j^{-\beta q} 2^{j s q} \sum_{\left(j_{1}, j_{2}\right) \in \Gamma_{j}(\alpha)}\left\|\Delta_{j_{1}, j_{2}} f\right\|_{p}^{q}\right)^{1 / q} .
$$

Then the embeddings of Theorem 4.4 can be rewritten in the following manner:

- if $q<\infty$,

$$
\begin{aligned}
& \mathcal{E}_{p, q,|\log |^{\beta-r_{1} / q}}^{s, \alpha}\left(\mathbb{R}^{2}\right) \hookrightarrow B_{p, q,|\log |^{\beta}}^{s, \alpha}\left(\mathbb{R}^{2}\right) \hookrightarrow \mathcal{E}_{p, q,|\log | \beta+r_{2} / q}^{s, \alpha}\left(\mathbb{R}^{2}\right) . \\
& \text { with } r_{1}= \begin{cases}q(1 / p-1)+\max (q-1,0) & \text { if } p \leq 1, \\
\max \left(q / p_{*}-1,0\right) & \text { if } p>1,\end{cases} \\
& \text { and } r_{2}= \begin{cases}1 & \text { if } p<1, \\
\max \left(1-q / p^{*}, 0\right) & \text { if } p \geq 1 .\end{cases}
\end{aligned}
$$

- if $q=\infty$,

$$
\mathcal{E}_{p, \infty,|\log |^{\beta-\max (1 / p-1,0)-1}}^{s, \alpha}\left(\mathbb{R}^{2}\right) \hookrightarrow B_{p, \infty,|\log |^{\beta}}^{s, \alpha}\left(\mathbb{R}^{2}\right) \hookrightarrow \mathcal{E}_{p, q,|\log |^{\beta}}^{s, \alpha}\left(\mathbb{R}^{2}\right) .
$$

In Proposition 4.7, an hyperbolic wavelet characterization of the spaces $\mathcal{E}_{p, q}^{s, \alpha}\left(\mathbb{R}^{2}\right)$ is given. Combining Proposition 4.7 and Theorem 4.4 directly implies Theorem 2.6.

Proposition 4.7. Let $(p, q) \in(0,+\infty]^{2}$, and let $s, \beta \in \mathbb{R}^{2}$. The following assertions are equivalent:

(1) $f \in \mathcal{E}_{p, q,|\log |^{\beta}}^{s, \alpha}\left(\mathbb{R}^{2}\right)$.

(2) $\left(\sum_{j \geq 0} j^{-\beta q} 2^{j s q} \sum_{\left(j_{1}, j_{2}\right) \in \Gamma_{j}} 2^{-\left(j_{1}+j_{2}\right) q / p} D_{j_{1}, j_{2}}\right)^{1 / q}<+\infty$. 
(3) $\left(\sum_{\left(j_{1}, j_{2}\right) \in \mathbb{N}_{0}^{2}}\left(\max \left(\frac{j_{1}}{\alpha_{1}}, \frac{j_{2}}{\alpha_{2}}\right)\right)^{-\beta q} 2^{\left(\max \left(j_{1} / \alpha_{1}, j_{2} / \alpha_{2}\right) s-\left(j_{1}+j_{2}\right) / p\right) q} D_{j_{1}, j_{2}}\right)^{1 / q}<+\infty$, where $D_{j_{1}, j_{2}}=\left(\sum_{\left(k_{1}, k_{2}\right) \in \mathbb{Z}^{2}}\left|c_{j_{1}, j_{2}, k_{1}, k_{2}}\right|^{p}\right)^{q / p}$.

We prove Proposition 4.7. Assertions (2) and (3) are equivalent because, for any $\left(j_{1}, j_{2}\right) \in \Gamma_{j}(\alpha)$, one has

$$
\max \left(\frac{j_{1}}{\alpha_{1}}, \frac{j_{2}}{\alpha_{2}}\right)+2-2 \leq j \leq \max \left(\frac{j_{1}}{\alpha_{1}}, \frac{j_{2}}{\alpha_{2}}\right)+2
$$

and $\cup \Gamma_{j}=(\mathbb{N} \cup\{-1\})^{2}$. The crucial point is the equivalence of the assertions (1) and (2).

Proof of the implication $(1) \Rightarrow(2)$ of Proposition 4.7. The proof of this implication relies on the following sampling lemma, which is an adaptation of Lemma 2.4 of [25] in the case of rectangular support.

Lemma 4.8. Let $p \in(0,+\infty]$ and $j=\left(j_{1}, j_{2}\right) \in \mathbb{N}_{0}^{2}$. Suppose $g \in \mathcal{S}^{\prime}\left(\mathbb{R}^{2}\right)$ and $\operatorname{supp}(\widehat{g}) \subset\left\{\xi,\left|\xi_{1}\right| \leq 2^{j_{1}+1}\right.$ and $\left.\left|\xi_{2}\right| \leq 2^{j_{2}+1}\right\}$. Then there exists $C>0$ such that

$$
\left(\sum_{\left(k_{1}, k_{2}\right) \in \mathbb{Z}^{2}} 2^{-\left(j_{1}+j_{2}\right)}\left|g\left(\frac{k_{1}}{2^{j_{1}}}, \frac{k_{2}}{2^{j_{2}}}\right)\right|^{p}\right)^{1 / p} \leq C\|g\|_{L^{p}}
$$

Proof. Let $\psi \in \mathcal{S}\left(\mathbb{R}^{2}\right)$ be such that $\operatorname{supp}(\widehat{\psi}) \subset\left\{\xi, \max \left(\left|\xi_{1}\right|,\left|\xi_{2}\right|\right) \leq \pi\right\}$ and $\widehat{\psi} \equiv 1$ on $[-2,2]^{2}$. Set $\psi_{j}(x)=2^{j_{1}+j_{2}} \psi\left(2^{j_{1}} x_{1}, 2^{j_{2}} x_{2}\right)$. One has

$$
\widehat{\psi}_{j} \equiv 1 \quad \text { on } \quad\left[-2^{j_{1}+1}, 2^{j_{1}+1}\right] \times\left[-2^{j_{2}+1}, 2^{j_{2}+1}\right] .
$$

By assumption $\operatorname{supp}(\widehat{g}) \subset\left[-2^{j_{1}+1}, 2^{j_{1}+1}\right] \times\left[-2^{j_{2}+1}, 2^{j_{2}+1}\right]$, so that, for any $x=\left(x_{1}, x_{2}\right) \in \mathbb{R}^{2}$ and any fixed $y=\left(y_{1}, y_{2}\right) \in \mathbb{R}^{2}$,

$$
g(x+y)=\left(\psi_{j} \star g\right)(x+y)=(2 \pi)^{-2} \int_{\xi_{1}=-2^{j_{1}+1}}^{2^{j_{1}+1}} \int_{\xi_{2}=-2^{j_{2}+1}}^{2^{j_{2}+1}} \widehat{\psi}_{j}(\xi) \widehat{g}(\xi) \mathrm{e}^{\mathrm{i} x \cdot \xi} \mathrm{e}^{\mathrm{i} y \cdot \xi} \mathrm{d} \xi
$$

Denote by $\widehat{h}_{j}$ the periodic extension of $\widehat{\psi}_{j}$ with period $2^{j_{i}+1} \pi$ in each variable $\xi_{i}(i=1,2)$. One has

$$
g(x+y)=(2 \pi)^{-2} \int_{\xi_{1}=-2^{j_{1}+1}}^{2^{j_{1}+1}} \int_{\xi_{2}=-2^{j_{2}+1}}^{2^{j_{2}+1}}\left(\widehat{h}_{j}(\xi) \mathrm{e}^{\mathrm{i} x \cdot \xi}\right)\left(\widehat{g}(\xi) \mathrm{e}^{\mathrm{i} y \cdot \xi}\right) \mathrm{d} \xi .
$$

Using an expansion of $\widehat{h}_{j} \mathrm{e}^{\mathrm{i} x \cdot \xi}$ in two-dimensional Fourier series, with $\tau=\left(\tau_{1}, \tau_{2}\right)$, 
it results:

$$
\begin{aligned}
& \widehat{h}_{j}(\xi) \mathrm{e}^{\mathrm{i} x \cdot \xi} \sum_{\left(\ell_{1}, \ell_{2}\right) \in \mathbb{Z}^{2}}\left(\int_{\tau_{1}=-2^{j_{1}+1} \pi}^{2^{j_{1}+1} \pi} \int_{\tau_{2}=-2^{j_{2}+1} \pi}^{2^{j_{2}+1} \pi} \widehat{h}_{j}(\tau) \mathrm{e}^{\mathrm{i} x \cdot \tau} \mathrm{e}^{-\mathrm{i}\left(2^{-j_{1}} \ell_{1} \tau_{1}+2^{-j_{2}} \ell_{2} \tau_{2}\right.} \mathrm{d} \tau_{1} \mathrm{~d} \tau_{2}\right) \\
& \times \mathrm{e}^{\mathrm{i}\left(2^{-j_{1}} \ell_{1} \xi_{1}+2^{-j_{2}} \ell_{2} \xi_{2}\right)} \\
&=\sum_{\left(\ell_{1}, \ell_{2}\right) \in \mathbb{Z}^{2}}\left(\int_{\tau_{1}=-2^{j_{1}+1} \pi}^{2^{j_{1}+1} \pi} \int_{\tau_{2}=-2^{j_{2}+1} \pi}^{2^{j_{2}+1} \pi} \widehat{\psi}_{j}(\tau) \mathrm{e}^{\mathrm{i} x \cdot \tau} \mathrm{e}^{-\mathrm{i} 2^{-j_{1}} \ell_{1} \tau_{1}} \mathrm{e}^{\left.-\mathrm{i} 2^{-j_{2} \ell_{2} \tau_{2}} \mathrm{~d} \tau_{1} \mathrm{~d} \tau_{2}\right)}\right. \\
& \quad \times \mathrm{e}^{\mathrm{i} 2^{-j_{1}} \ell_{1} \xi_{1}} \mathrm{e}^{\mathrm{i} 2^{-j_{2}} \ell_{2} \xi_{2}} \\
&=2^{-\left(j_{1}+j_{2}\right)} \sum_{\left(\ell_{1}, \ell_{2}\right) \in \mathbb{Z}^{2}} \psi_{j}\left(x-2^{-j} \ell\right) \mathrm{e}^{\mathrm{i} 2^{-j_{1}} \ell_{1} \xi_{1}} \mathrm{e}^{\mathrm{i} 2^{-j_{2} \ell_{2} \xi_{2}},}
\end{aligned}
$$

where for $j=\left(j_{1}, j_{2}\right)$ and $\ell=\left(\ell_{1}, \ell_{2}\right)$, the notation $2^{-j} \ell=\left(2^{-j_{1}} \ell_{1}, 2^{-j_{2}} \ell_{2}\right)$ is used. Replacing $\widehat{h}_{j}(\xi) \mathrm{e}^{\mathrm{i} x \cdot \xi}$ with the last sum in equation (4.14) yields that, for any $x=\left(x_{1}, x_{2}\right) \in \mathbb{R}^{2}$ and any fixed $y=\left(y_{1}, y_{2}\right) \in \mathbb{R}^{2}$,

$$
\begin{aligned}
& g(x+y) \\
& =\frac{2^{-\left(j_{1}+j_{2}\right)}}{4 \pi^{2}} \sum_{\left(\ell_{1}, \ell_{2}\right) \in \mathbb{Z}^{2}}\left(\int_{\xi_{1}=-2^{j_{1}+1}}^{2^{j_{1}+1}} \int_{\xi_{2}=-2^{j_{2}+1}}^{2^{j_{2}+1}} \psi_{j}\left(x-2^{-j} \ell\right) \mathrm{e}^{\mathrm{i} 2^{-j} \ell \cdot \xi}\left(\widehat{g}(\xi) \mathrm{e}^{\mathrm{i} y \cdot \xi}\right) \mathrm{d} \xi\right) \\
& =2^{-\left(j_{1}+j_{2}\right)} \sum_{\left(\ell_{1}, \ell_{2}\right) \in \mathbb{Z}^{2}} g\left(2^{-j} \ell+y\right) \psi_{j}\left(x-2^{-j} \ell\right) .
\end{aligned}
$$

Hence, for all $y \in \lambda_{j_{1}, j_{2}, k_{1}, k_{2}}=\left[2^{-j_{1}} k_{1}, 2^{-j_{1}}\left(k_{1}+1\right)\right) \times\left[2^{-j_{2}} k_{2}, 2^{-j_{2}}\left(k_{2}+1\right)\right)$,

$$
\begin{aligned}
& \quad \sup _{\left|z_{1}-2^{-j_{1}} k_{1}\right| \leq 2^{-j_{1}},\left|z_{2}-2^{-j_{2}} k_{2}\right| \leq 2^{-j_{2}}}|g(z)| \\
& \quad \leq \sup _{\left|x_{1}\right| \leq 2^{-j_{1}} \sqrt{2},\left|x_{2}\right| \leq 2^{-j_{2} \sqrt{2}}|g(x+y)|}\left|g\left(2^{-j} \ell+y\right)\right| \cdot \sup _{\max \left(2^{j_{1}}\left|x_{1}\right|, 2^{j_{2}}\left|x_{2}\right|\right) \leq \sqrt{2}}\left|\psi_{j}\left(x-2^{-j} \ell\right)\right| \\
& \quad \leq 2^{-\left(j_{1}+j_{2}\right)} \sum_{\left(\ell_{1}, \ell_{2}\right) \in \mathbb{Z}^{2}}\left|2^{-\left(j_{1}+j_{2}\right)} \sum_{\left(\ell_{1}, \ell_{2}\right) \in \mathbb{Z}^{2}}\right| g\left(2^{-j} \ell+y\right) \mid \cdot \frac{1}{(1+|\ell|)^{M}},
\end{aligned}
$$

where the last inequality follows from the fast decay of $\psi$. Take $M$ sufficiently large and use either the $\mathrm{p}$ triangular inequality or the Hölder inequality as $p \in(0,1)$ or $p \in[1,+\infty]$. Hence, one has

$$
\begin{aligned}
\left|g\left(2^{-j_{1}} k_{1}, 2^{-j_{2}} k_{2}\right)\right|^{p} & \leq \sup _{\left|z_{1}-2^{-j_{1}} k_{1}\right| \leq 2^{-j_{1}},\left|z_{2}-2^{-j_{2}} k_{2}\right| \leq 2^{-j_{2}}}|g(z)|^{p} \\
& \leq C 2^{-\left(j_{1}+j_{2}\right)} \sum_{\left(\ell_{1}, \ell_{2}\right) \in \mathbb{Z}^{2}}\left|g\left(2^{-j} \ell+y\right)\right|^{p} \cdot \frac{1}{(1+|\ell|)^{M^{\prime}}},
\end{aligned}
$$


for some $M^{\prime}>1$. Integration over $y \in \lambda_{j_{1}, j_{2}, k_{1}, k_{2}}$ leads to

$$
2^{-\left(j_{1}+j_{2}\right)}\left|g\left(2^{-j_{1}} k_{1}, 2^{-j_{2}} k_{2}\right)\right|^{p} \leq \sum_{\left(\ell_{1}, \ell_{2}\right) \in \mathbb{Z}^{2}} \frac{1}{(1+|\ell|)^{M^{\prime}}} \int_{\lambda_{j_{1}, j_{2}, k_{1}, k_{2}}}|g(y)|^{p} \mathrm{~d} y .
$$

Summing over $k \in \mathbb{Z}^{2}$ gives

$$
\sum_{k} 2^{-\left(j_{1}+j_{2}\right)}\left|g\left(2^{-j_{1}} k_{1}, 2^{-j_{2}} k_{2}\right)\right|^{p} \leq \sum_{k} \sum_{\left(\ell_{1}, \ell_{2}\right) \in \mathbb{Z}^{2}} \frac{1}{(1+|\ell|)^{3}} \int_{\lambda_{j_{1}, j_{2}, k_{1}, k_{2}}}|g(y)|^{p} \mathrm{~d} y,
$$

which concludes the proof of Lemma 4.8.

Now, observe that $c_{j_{1}, j_{2}, k_{1}, k_{2}}=\Delta_{j_{1}, j_{2}} f\left(2^{-j_{1}} k_{1}, 2^{-j_{2}} k_{2}\right)$. By Lemma 4.8 applied to $g=\Delta_{j_{1}, j_{2}} f \in \mathcal{S}\left(\mathbb{R}^{2}\right)$, one has

$$
\sum_{\left(k_{1}, k_{2}\right) \in \mathbb{Z}^{2}}\left|c_{j_{1}, j_{2}, k_{1}, k_{2}}\right|^{p}=\sum_{\left(k_{1}, k_{2}\right) \in \mathbb{Z}^{2}}\left|\Delta_{j_{1}, j_{2}} f\left(2^{-j_{1}} k_{1}, 2^{-j_{2}} k_{2}\right)\right|^{p} \leq C 2^{j_{1}} 2^{j_{2}}\left\|\Delta_{j_{1}, j_{2}} f\right\|_{p}^{p},
$$

which is the desired wavelet characterization.

Proof of the implication $(2) \Rightarrow(1)$ of Proposition 4.7. To obtain the converse implication, the approach of the proof of Theorem 3.1 of [25] is followed.

Since $\phi_{j_{1}, j_{2}}$ and $\psi_{m_{1}, m_{2}, k_{1}, k_{1}}$ are both defined as tensor products, Lemma 3.3 of [25] can be applied to conclude that there exists $C>0$ such that for any $\alpha>0$ and for all $x=\left(x_{1}, x_{2}\right) \in \mathbb{R}^{2}$ one has

$$
\begin{aligned}
& \left|\phi_{j_{1}, j_{2}} \star \psi_{m_{1}, m_{2}, k_{1}, k_{1}}(x)\right| \\
& \quad \leq \frac{C 2^{-\left(\left|j_{1}-m_{1}\right|+\left|j_{2}-m_{2}\right|\right)(M+3)}}{\left(1+2^{\inf \left(j_{1}, m_{1}\right)}\left|x_{1}-2^{-m_{1}} k_{1}\right|\right)^{\alpha}\left(1+2^{\inf \left(j_{2}, m_{2}\right)}\left|x_{2}-2^{-m_{2}} k_{2}\right|\right)^{\alpha}},
\end{aligned}
$$

where $M$ denotes the number of vanishing moments of the wavelets.

Lemma 4.9 is analogous to Lemma 3.4 of [25].

Lemma 4.9. Let $p \in[1,+\infty]$, and let $\ell_{1}, \ell_{2}, m_{1}$, and $m_{2}$ be integers such that $\ell_{1} \leq m_{1}$ and $\ell_{2} \leq m_{2}$. Additionally suppose given functions $g_{k_{1}, k_{2}}$ satisfying, $\forall x=\left(x_{1}, x_{2}\right) \in \mathbb{R}^{2}$, the inequality

$$
\left|g_{k_{1}, k_{2}}(x)\right| \leq \frac{C}{\left(1+2^{\ell_{1}}\left|x_{1}-2^{-m_{1}} k_{1}\right|\right)^{2}\left(1+2^{\ell_{2}}\left|x_{2}-2^{-m_{2}} k_{2}\right|\right)^{2}}
$$

for some $C>0$. Set

$$
F=\sum_{k=\left(k_{1}, k_{2}\right) \in \mathbb{Z}^{2}} d_{k_{1}, k_{2}} g_{k_{1}, k_{2}}
$$

Then

$$
\|F\|_{L^{p}} \leq C 2^{-\left(m_{1}+m_{2}\right) / p} 2^{m_{1}-\ell_{1}} 2^{m_{2}-\ell_{2}} \cdot\left(\sum_{k=\left(k_{1}, k_{2}\right) \in \mathbb{Z}^{2}}\left|d_{k_{1}, k_{2}}\right|^{p}\right)^{1 / p}
$$


Proof. By definition of the $L^{p}$-norm, one has

$$
\begin{aligned}
\|F\|_{L^{p}}^{p} & =\int_{\mathbb{R}^{2}}\left|\sum_{k=\left(k_{1}, k_{2}\right) \in \mathbb{Z}^{2}} d_{k_{1}, k_{2}} g_{k_{1}, k_{2}}(x)\right|^{p} \mathrm{~d} x \\
& \leq \sum_{k^{\prime}=\left(k_{1}^{\prime}, k_{2}^{\prime}\right) \in \mathbb{Z}^{2}} \int_{\lambda_{m_{1}, m_{2}, k_{1}^{\prime}, k_{2}^{\prime}}}\left|\sum_{k=\left(k_{1}, k_{2}\right) \in \mathbb{Z}^{2}} d_{k_{1}, k_{2}} g_{k_{1}, k_{2}}(x)\right|^{p} \mathrm{~d} x
\end{aligned}
$$

where the hyperbolic dyadic cubes $\lambda_{m_{1}, m_{2}, k_{1}^{\prime}, k_{2}^{\prime}}$ are defined in (3.3). Observe now that, by the usual triangle inequality and by inequality (4.16), there exists $C>0$ such that, for any $\left(k_{1}, k_{2}\right) \in \mathbb{Z}^{2},\left(k_{1}^{\prime}, k_{2}^{\prime}\right) \in \mathbb{Z}^{2}$,

$$
\begin{aligned}
\sup _{x \in \lambda_{m_{1}, m_{2}, k_{1}^{\prime}, k_{2}^{\prime}}} & \left|\sum_{\left(k_{1}, k_{2}\right) \in \mathbb{Z}^{2}} d_{k_{1}, k_{2}} g_{k_{1}, k_{2}}(x)\right| \\
\leq & \sum_{\left(k_{1}, k_{2}\right) \in \mathbb{Z}^{2}} \frac{\left|d_{k_{1}, k_{2}}\right|}{\prod_{i=1,2}\left(1+2^{\ell_{i}}\left|2^{-m_{i}} k_{i}^{\prime}-2^{-m_{i}} k_{i}\right|\right)^{2}}=I\left(m_{1}, m_{2}, k_{1}^{\prime}, k_{2}^{\prime}\right) .
\end{aligned}
$$

Hence one has

$$
\|F\|_{L^{p}}^{p} \leq C 2^{-\left(m_{1}+m_{2}\right)} \sum_{\left(k_{1}^{\prime}, k_{2}^{\prime}\right) \in \mathbb{Z}^{2}} I\left(m_{1}, m_{2}, k_{1}^{\prime}, k_{2}^{\prime}\right)^{p} .
$$

Recall the usual convolution inequality

$$
\left\|s * s^{\prime}\right\|_{\ell_{p}\left(\mathbb{Z}^{2}\right)}^{p} \leq\|s\|_{\ell_{p}\left(\mathbb{Z}^{2}\right)}^{p}\left\|s^{\prime}\right\|_{\ell^{1}\left(\mathbb{Z}^{2}\right)}^{p},
$$

valid for any sequences $s, s^{\prime}$ in $\ell^{p}\left(\mathbb{Z}^{2}\right)$ for $p \geq 1$. Applied to $s=\left|d_{k_{1}, k_{2}}\right|$ and $s^{\prime}=\left(1+2^{\ell_{1}-m_{1}}\left|k_{1}^{\prime}-k_{1}\right|\right)^{-2}\left(1+2^{\ell_{2}-m_{2}}\left|k_{2}^{\prime}-k_{2}\right|\right)^{-2}$, this gives

$$
\begin{aligned}
\|F\|_{L^{p}}^{p} \leq & C 2^{-\left(m_{1}+m_{2}\right)}\left(\sum_{\left(k_{1}, k_{2}\right) \in \mathbb{Z}^{2}}\left|d_{k_{1}, k_{2}}\right|^{p}\right) \\
& \times\left(\sum_{\left(k_{1}^{\prime}, k_{2}^{\prime}\right) \in \mathbb{Z}^{2}} \frac{1}{\left(1+2^{\ell_{1}-m_{1}}\left|k_{1}^{\prime}\right|\right)^{2}\left(1+2^{\ell_{2}-m_{2}}\left|k_{2}^{\prime}\right|\right)^{2}}\right)^{p} .
\end{aligned}
$$

Recall the classical inequality

$$
\sum_{k^{\prime}=\left(k_{1}^{\prime}, k_{2}^{\prime}\right) \in \mathbb{Z}^{2}} \frac{1}{\left(1+2^{\ell_{1}-m_{1}}\left|k_{1}^{\prime}\right|\right)^{2}\left(1+2^{\ell_{2}-m_{2}}\left|k_{2}^{\prime}\right|\right)^{2}} \leq C 2^{m_{1}-\ell_{1}} 2^{m_{2}-\ell_{2}} .
$$

Hence

$$
\begin{aligned}
\|F\|_{L^{p}}^{p} \leq & C 2^{-\left(m_{1}+m_{2}\right)} 2^{\left(m_{1}-\ell_{1}\right) p} 2^{\left(m_{2}-\ell_{2}\right) p}\left(\sum_{k=\left(k_{1}, k_{2}\right) \in \mathbb{Z}^{2}}\left|d_{k_{1}, k_{2}}\right|^{p}\right) \\
& \times\left(\sum_{k^{\prime}=\left(k_{1}^{\prime}, k_{2}^{\prime}\right) \in \mathbb{Z}^{2}} \frac{1}{\prod_{i=1,2}\left(1+2^{\ell_{i}-m_{i}}\left|k_{i}^{\prime}\right|\right)^{2}}\right)^{p},
\end{aligned}
$$

which directly yields the required result. This concludes the proof of Lemma 4.9. 
Now we return to implication $(2) \Rightarrow(1)$ of Proposition 4.7. Two cases are considered: $p \in(0,1)$ and $p \in[1,+\infty]$.

First we assume that $p \in(0,1)$. We need to bound $\left\|\Delta_{j_{1}, j_{2}} f\right\|_{L^{p}}=\left\|\phi_{j_{1}, j_{2}} \star f\right\|_{L^{p}}$. Observe that

$$
\phi_{j_{1}, j_{2}} \star f=\sum_{m_{1}, m_{2}} \sum_{k_{1}, k_{2}} c_{m_{1}, m_{2}, k_{1}, k_{2}}\left(\phi_{j_{1}, j_{2}} \star \psi_{m_{1}, m_{2}, k_{1}, k_{2}}\right) .
$$

By the $p$-triangular inequality, there results

$$
\forall x \in \mathbb{R}^{2},\left|\phi_{j_{1}, j_{2}} \star f(x)\right|^{p} \leq \sum_{m_{1}, m_{2}} \sum_{k_{1}, k_{2}}\left|c_{m_{1}, m_{2}, k_{1}, k_{2}}\right|^{p}\left|\left(\phi_{j_{1}, j_{2}} \star \psi_{m_{1}, m_{2}, k_{1}, k_{2}}\right)(x)\right|^{p} .
$$

By the inequality (4.15), for all $x=\left(x_{1}, x_{2}\right) \in \mathbb{R}^{2}$, one has

$$
\begin{aligned}
\left|\phi_{j_{1}, j_{2}} \star f(x)\right|^{p} \leq & \sum_{m_{1}, m_{2}} \sum_{k_{1}, k_{2}}\left|c_{m_{1}, m_{2}, k_{1}, k_{2}}\right|^{p} \\
& \times \frac{2^{-p\left(\left|j_{1}-m_{1}\right|+\left|j_{2}-m_{2}\right|\right)(M+3)}}{\left(\left(1+2^{\inf \left(j_{1}, m_{1}\right)}\left|x_{1}-2^{-m_{1}} k_{1}\right|\right)\left(1+2^{\inf \left(j_{2}, m_{2}\right)}\left|x_{2}-2^{-m_{2}} k_{2}\right|\right)\right)^{p \alpha}} .
\end{aligned}
$$

Integration over $\mathbb{R}^{2}$ implies that

$$
\left\|\phi_{j_{1}, j_{2}} \star f(x)\right\|_{L^{p}}^{p} \leq \sum_{m_{1}, m_{2}} \sum_{k_{1}, k_{2}}\left|c_{m_{1}, m_{2}, k_{1}, k_{2}}\right|^{p} 2^{-p\left(\left|j_{1}-m_{1}\right|+\left|j_{2}-m_{2}\right|\right)(M+3)} .
$$

Hence

$$
\begin{aligned}
& \|f\|_{\mathcal{E}_{p, q,|\log | \beta}^{s, \alpha}}^{q}=\sum_{j_{1}, j_{2}}\left(\max \left(\frac{j_{1}}{\alpha_{1}}, \frac{j_{2}}{\alpha_{2}}\right)\right)^{-\beta q} 2^{q s \max \left(j_{1} / \alpha_{1}, j_{2} / \alpha_{2}\right)}\left\|\phi_{j_{1}, j_{2}} \star f(x)\right\|_{L^{p}}^{q}
\end{aligned}
$$

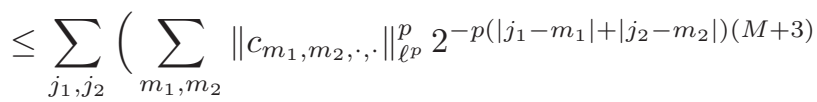

$$
\begin{aligned}
& \left.\times\left(\max \left(\frac{j_{1}}{\alpha_{1}}, \frac{j_{2}}{\alpha_{2}}\right)\right)^{-\beta p} 2^{p s \max \left(j_{1} / \alpha_{1}, j_{2} / \alpha_{2}\right)}\right)^{q / p} .
\end{aligned}
$$

For any $t \in \mathbb{R}$, set $(t)_{+}=\max (t, 0)$ and define

$$
\operatorname{sgn}(t)=\left\{\begin{aligned}
1 & \text { if } t>0 \\
0 & \text { if } t=0 \\
-1 & \text { if } t<0
\end{aligned}\right.
$$

Now observe now that, for any integers $j$ and $m$,

$$
m-(m-j)_{+} \leq j \leq(j-m)_{+}+m,
$$

and that, for any integers $j_{1}, j_{2}, m_{1}$, and $m_{2}$,

$$
\frac{\max \left(m_{1} / \alpha_{1}, m_{2} / \alpha_{2}\right)}{1-\frac{\max \left(\left(m_{1}-j_{1}\right)_{+} / \alpha_{1},\left(m_{2}-j_{2}\right)_{+} / \alpha_{2}\right)}{\max \left(m_{1} / \alpha_{1}, m_{2} / \alpha_{2}\right)}} \leq \max \left(\frac{j_{1}}{\alpha_{1}}, \frac{j_{2}}{\alpha_{2}}\right)
$$


and

$$
\max \left(\frac{j_{1}}{\alpha_{1}}, \frac{j_{2}}{\alpha_{2}}\right) \leq \max \left(\frac{m_{1}}{\alpha_{1}}, \frac{m_{2}}{\alpha_{2}}\right)\left[1+\max \left(\frac{\left(j_{1}-m_{1}\right)_{+}}{\alpha_{1}}, \frac{\left(j_{2}-m_{2}\right)_{+}}{\alpha_{2}}\right)\right]
$$

(except in the case $m_{1}=m_{2}=0$ which can be treated separately). Hence

$$
\|f\|_{\mathcal{E}_{p, q,|\log | \beta}^{s, \alpha}}^{q} \leq \sum_{j_{1}, j_{2}}\left(\sum_{m_{1}, m_{2}} u_{m_{1}, m_{2}} v_{j_{1}-m_{1}, j_{2}-m_{2}} \cdot\right)^{q / p},
$$

with

$$
u_{m_{1}, m_{2}}=\left(\max \left(\frac{m_{1}}{\alpha_{1}}, \frac{m_{2}}{\alpha_{2}}\right)\right)^{-\beta p} 2^{p s \max \left(m_{1} / \alpha_{1}, m_{2} / \alpha_{2}\right)} \| c_{m_{1}, m_{2}, \cdot, \cdot \|_{\ell^{p}}^{p}}
$$

and

$v_{j_{1}, j_{2}}=2^{-p\left(\left|j_{1}\right|+\left|j_{2}\right|\right)(M+3)}\left[1+\max \left(\frac{\left(j_{1}\right)_{+}}{\alpha_{1}}, \frac{\left(j_{2}\right)_{+}}{\alpha_{2}}\right)\right]^{-\beta p} 2^{\operatorname{sgn}(s) p s \max \left(\left(j_{1}\right)_{+} / \alpha_{1},\left(j_{2}\right)_{+} / \alpha_{2}\right)}$.

If $q / p>1$, Young's inequality can be applied. It states that, for any sequences $s$ and $s^{\prime}$,

$$
\left\|s * s^{\prime}\right\|_{\ell^{q / p}\left(\mathbb{Z}^{2}\right)} \leq\|s\|_{\ell^{q / p}\left(\mathbb{Z}^{2}\right)}\left\|s^{\prime}\right\|_{\ell^{1}\left(\mathbb{Z}^{2}\right)},
$$

whereas if $q / p \leq 1$ the usual $(q / p)$-triangle inequality and the usual inequality $\left\|s * s^{\prime}\right\|_{\ell^{1}\left(\mathbb{Z}^{2}\right)} \leq\|s\|_{\ell^{1}\left(\mathbb{Z}^{2}\right)}\left\|s^{\prime}\right\|_{\ell^{1}\left(\mathbb{Z}^{2}\right)}$ valid for any sequence $s$ and $s^{\prime}$ can applied. In either case, there is obtained the inequality

$$
\begin{gathered}
\|f\|_{\mathcal{E}_{p, q,|\log | \beta}^{s, \alpha}}^{q} \leq\left(\sum_{m_{1}, m_{2}}\left(\max \left(\frac{m_{1}}{\alpha_{1}}, \frac{m_{2}}{\alpha_{2}}\right)\right)^{-\beta p} 2^{q s \max \left(m_{1} / \alpha_{1}, m_{2} / \alpha_{2}\right)} \| c_{m_{1}, m_{2}, \cdot,\left.\cdot\right|_{\ell^{p}}}^{p}\right) \\
\quad \times \sum_{j_{1}, j_{2}}\left(2^{-p\left(j_{1}+j_{2}\right)(M+3)}\left(\max \left(\frac{j_{1}}{\alpha_{1}}, \frac{j_{2}}{\alpha_{2}}\right)\right)^{-\beta p} 2^{p s \max \left(j_{1} / \alpha_{1}, j_{2} / \alpha_{2}\right)}\right)^{\max (q / p, 1)} .
\end{gathered}
$$

If the wavelets have sufficiently many vanishing moments, we get that

$$
\|f\|_{\mathcal{E}_{p, q,|\log |^{\beta}}^{q, \alpha}}^{q} \leq C\left(\sum_{m_{1}, m_{2}}\left(\max \left(\frac{m_{1}}{\alpha_{1}}, \frac{m_{2}}{\alpha_{2}}\right)\right)^{-\beta p} 2^{q s \max \left(m_{1} / \alpha_{1}, m_{2} / \alpha_{2}\right)} \| c_{m_{1}, m_{2}, \cdot, \cdot||_{\ell^{p}}^{p}}\right),
$$

which is the required result.

Now we consider the case $p \in[1,+\infty]$. In this case, observe that

$$
\Delta_{j_{1}, j_{2}} f=\sum_{k_{1}, k_{2}} d_{k_{1}, k_{2}} g_{k_{1}, k_{2}}
$$

with

and

$$
g_{k_{1}, k_{2}}=2^{\left(\left|j_{1}-m_{1}\right|+\left|j_{2}-m_{2}\right|\right)(M+3)}\left(\phi_{j_{1}, j_{2}} \star \psi_{m_{1}, m_{2}, k_{1}, k_{2}}\right),
$$

$$
d_{k_{1}, k_{2}}=2^{-\left(\left|j_{1}-m_{1}\right|+\left|j_{2}-m_{2}\right|\right)(M+3)} c_{j_{1}, j_{2}, k_{1}, k_{2}} .
$$

We set $\ell_{1}=\inf \left(j_{1}, m_{1}\right)$ and $\ell_{2}=\inf \left(j_{2}, m_{2}\right)$. Lemma 4.9 gives

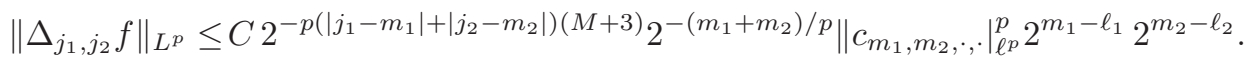

Again the two cases $q \leq 1$ and $q>1$ are distinguished and the same approach as in the case $p \in(0,1)$ is followed. It leads to the required conclusion. 


\subsection{Proof of Theorem 3.4}

First a two-microlocal criterion is proved.

Proposition 4.10. 1) Assume that $f \in \mathcal{C}^{s, \alpha}\left(x_{0}\right)$. Then there exists $C>0$ such that, for any $\left(j_{1}, j_{2}, k_{1}, k_{2}\right) \in(\mathbb{N} \cup\{-1\})^{2} \times \mathbb{Z}^{2}$,

(4.18) $\left|c_{j_{1}, j_{2}, k_{1}, k_{2}}\right| \leq C \min \left(2^{-j_{1} s / \alpha_{1}}+\left|\frac{k_{1}}{2^{j_{1}}}-a\right|^{s / \alpha_{1}}, 2^{-j_{2} s / \alpha_{2}}+\left|\frac{k_{2}}{2^{j_{2}}}-b\right|^{s / \alpha_{2}}\right)$.

2) Conversely, if $f$ is uniformly Hölder and (4.18) holds, then $f \in \mathcal{C}_{|\log |^{2}}^{s, \alpha}\left(x_{0}\right)$.

Proof. First assume that $f \in \mathcal{C}^{s, \alpha}\left(x_{0}\right)$ with $x_{0}=(a, b)$. Assume that $j_{1} \neq-1$ and $j_{2} \neq-1$. By definition of the hyperbolic wavelet coefficients one has

$$
c_{j_{1}, j_{2}, k_{1}, k_{2}}=2^{j_{1}+j_{2}} \int_{\mathbb{R}^{2}} f\left(x_{1}, x_{2}\right) \psi\left(2^{j_{1}} x_{1}-k_{1}\right) \psi\left(2^{j_{2}} x_{2}-k_{2}\right) \mathrm{d} x_{1} \mathrm{~d} x_{2} .
$$

Since $\psi$ admits at least one vanishing moment, there hold the equalities

$c_{j_{1}, j_{2}, k_{1}, k_{2}}=2^{j_{1}+j_{2}} \int_{\mathbb{R}^{2}}\left(f\left(x_{1}, x_{2}\right)-P_{x_{0}}\left(a, x_{2}\right)\right) \psi\left(2^{j_{1}} x_{1}-k_{1}\right) \psi\left(2^{j_{2}} x_{2}-k_{2}\right) \mathrm{d} x_{1} \mathrm{~d} x_{2}$,

$c_{j_{1}, j_{2}, k_{1}, k_{2}}=2^{j_{1}+j_{2}} \int_{\mathbb{R}^{2}}\left(f\left(x_{1}, x_{2}\right)-P_{x_{0}}\left(x_{1}, b\right)\right) \psi\left(2^{j_{1}} x_{1}-k_{1}\right) \psi\left(2^{j_{2}} x_{2}-k_{2}\right) \mathrm{d} x_{1} \mathrm{~d} x_{2}$.

Equality (4.19) and the assumption $f \in \mathcal{C}^{s, \alpha}\left(x_{0}\right)$ imply that

$$
\begin{aligned}
& \left|c_{j_{1}, j_{2}, k_{1}, k_{2}}\right| \leq 2^{j_{1}+j_{2}} \int\left|x_{1}-a\right|_{\alpha}^{s}\left|\psi\left(2^{j_{1}} x_{1}-k_{1}\right) \psi\left(2^{j_{2}} x_{2}-k_{2}\right)\right| \mathrm{d} x_{1} \mathrm{~d} x_{2} \\
& \leq 2^{j_{1}+j_{2}} \int_{\mathbb{R}^{2}}\left(\left|x_{1}-\frac{k_{1}}{2^{j_{1}}}\right|^{s / \alpha_{1}}+\left|\frac{k_{1}}{2^{j_{1}}}-a\right|^{s / \alpha_{1}}\right)\left|\psi\left(2^{j_{1}} x_{1}-k_{1}\right) \psi\left(2^{j_{2}} x_{2}-k_{2}\right)\right| \mathrm{d} x_{1} \mathrm{~d} x_{2} .
\end{aligned}
$$

We now set $u_{1}=2^{j_{1}} x_{1}-k_{1}, u_{2}=2^{j_{2}} x_{2}-k_{2}$ and deduce that

$$
\begin{aligned}
\left|c_{j_{1}, j_{2}, k_{1}, k_{2}}\right| \leq & \left(2^{-j_{1} s / \alpha_{1}} \int_{\mathbb{R}^{2}}\left|u_{1}\right|^{s / \alpha_{1}}\left|\psi\left(u_{1}\right) \psi\left(u_{2}\right)\right| \mathrm{d} u_{1} \mathrm{~d} u_{2}\right. \\
& \left.+\left|\frac{k_{1}}{2^{j_{1}}}-a\right|^{s / \alpha_{1}} \int\left|\psi\left(u_{1}\right) \psi\left(u_{2}\right)\right| \mathrm{d} u_{1} \mathrm{~d} u_{2}\right) .
\end{aligned}
$$

Hence for some $C$ depending only on $\psi, s$, and $\alpha$ one has

$$
\left|c_{j_{1}, j_{2}, k_{1}, k_{2}}\right| \leq C\left(2^{-j_{1} s / \alpha_{1}}+\left|\frac{k_{1}}{2^{j_{1}}}-a\right|^{s / \alpha_{1}}\right) .
$$

A similar approach yields that

$$
\left|c_{j_{1}, j_{2}, k_{1}, k_{2}}\right| \leq C\left(2^{-j_{2} s / \alpha_{2}}+\left|\frac{k_{2}}{2^{j_{2}}}-b\right|^{s / \alpha_{2}}\right) .
$$

This shows that (4.18) can be interpreted as a necessary condition for pointwise regularity. 
Now we prove the converse result. Assuming that (4.18) holds, the initial aim is to define a polynomial approximation of $f$ at $x_{0}$. To this end, a Taylor expansion is used to investigate the differentiability of $f$ at $x_{0}$. We define $f_{j}$ by

$$
f_{j}=\sum_{\left(j_{1}, j_{2}\right) \in \Gamma_{j}(\alpha)} \sum_{\left(k_{1}, k_{2}\right) \in \mathbb{Z}^{2}} c_{j_{1}, j_{2}, k_{1}, k_{2}} \psi_{j_{1}, j_{2}, k_{1}, k_{2}} .
$$

where the notation is the same as in the proof of Proposition 2.8. One has

$$
\begin{aligned}
\left|f_{j}(x)\right| \leq & \sum_{\left(j_{1}, j_{2}\right) \in \Gamma_{j}} \sum_{\left(k_{1}, k_{2}\right) \in \mathbb{Z}^{2}} \frac{\min \left(2^{-j_{1} s / \alpha_{1}}+\left|\frac{k_{1}}{2^{j_{1}}}-a\right|^{s / \alpha_{1}}, 2^{-j_{2} s / \alpha_{2}}+\left|\frac{k_{2}}{2^{j_{2}}}-a\right|^{s / \alpha_{2}}\right)}{\left(1+\left|2^{j_{1}} x_{1}-k_{1}\right|\right)^{N}\left(1+\left|2^{j_{2}} x_{2}-k_{2}\right|\right)^{N}} \\
\leq & \sum_{j_{1} \leq j} \sum_{k_{1}, k_{2}} \frac{2^{-j s}+\left|\frac{k_{2}}{2^{j}}-x_{2}\right|^{s / \alpha_{2}}+\left|x_{2}-b\right|^{s / \alpha_{2}}}{\left(1+\left|2^{j_{1}} x_{1}-k_{1}\right|\right)^{N}\left(1+\left|2^{j_{2}} x_{2}-k_{2}\right|\right)^{N}} \\
& +\sum_{j_{2} \leq j} \frac{2^{-j s}+\left|\frac{k_{1}}{2^{j_{1}}}-x_{1}\right|^{s / \alpha_{1}}+\left|x_{1}-a\right|^{s / \alpha_{2}}}{\left(1+\left|2^{j_{1}} x_{1}-k_{1}\right|\right)^{N}\left(1+\left|2^{j_{2}} x_{2}-k_{2}\right|\right)^{N}} .
\end{aligned}
$$

Then

$$
\left|f_{j}(x)\right| \leq C\left(j 2^{-j s}+j\left|x_{1}-a\right|^{s / \alpha_{1}}+j\left|x_{2}-b\right|^{s / \alpha_{2}}\right) .
$$

In the same way, if $\beta=\left(\beta_{1}, \beta_{2}\right)$, an upper bound of $\left|\partial^{\beta} f_{j}\right|$ is given by

$$
\sum_{\left(j_{1}, j_{2}\right) \in \Gamma_{j}} 2^{j_{1} \beta_{1}+j_{2} \beta_{2}} \sum_{\left(k_{1}, k_{2}\right) \in \mathbb{Z}^{2}} \frac{\min \left(2^{-j_{1} s / \alpha_{1}}+\left|\frac{k_{1}}{2^{j_{1}}}-a\right|^{s / \alpha_{1}}, 2^{-j_{2} s / \alpha_{2}}+\left|\frac{k_{2}}{2^{j_{2}}}-a\right|^{s / \alpha_{2}}\right)}{\left(1+\left|2^{j_{1}} x_{1}-k_{1}\right|\right)^{N}\left(1+\left|2^{j_{2}} x_{2}-k_{2}\right|\right)^{N}} .
$$

Then

$$
\left|\partial^{\beta} f_{j}(x)\right| \leq C 2^{j\left(\beta_{1} \alpha_{1}+\beta_{2} \alpha_{2}\right)}\left(2^{-j s}+\left|x_{1}-a\right|^{s / \alpha_{1}}+\left|x_{2}-b\right|^{s / \alpha_{2}}\right) .
$$

Hence, the function $f$ is $\beta$-differentiable at $x_{0}$ provided that $\beta_{1} \alpha_{1}+\beta_{2} \alpha_{2} \leq s$. The Taylor polynomial of $f$ at $x_{0}$ is given by

$$
P_{j, x_{0}}(x)=\sum_{\beta_{1} \alpha_{1}+\beta_{2} \alpha_{2} \leq s} \frac{\left(x-x_{0}\right)^{\beta}}{\beta !} \partial^{\beta} f_{j}\left(x_{0}\right)
$$

and

$$
P_{x_{0}}(x)=\sum_{j} P_{j, x_{0}}(x) .
$$

We shall now bound $\left|f(x)-P_{x_{0}}(x)\right|$ in a neighborhood of $x_{0}$. Recall that $f$ is assumed to be uniformly Hölder, namely there exists $\varepsilon_{0}^{*}>0$ such that $f \in \mathcal{C}^{\varepsilon_{0}^{*}}\left(\mathbb{R}^{2}\right)$. The inclusions between Hölder spaces with different anisotropies (see [48]) lead to the existence of $\varepsilon_{0}$ such that $f \in \mathcal{C}^{\varepsilon_{0}, \alpha}\left(\mathbb{R}^{2}\right)$. Set $J_{1}=\left[\alpha J / \varepsilon_{0}\right]$. Observe that $\left|f(x)-P_{x_{0}}(x)\right| \leq \sum_{j \leq J}\left|f_{j}(x)-P_{j, x_{0}}(x)\right|+\sum_{j=J+1}^{J_{1}}\left|f_{j}(x)\right|+\sum_{j>J_{1}}\left|f_{j}(x)\right|+\sum_{j>J}\left|P_{j, x_{0}}(x)\right|$.

Now we bound each term of the right hand side of this inequality. 
First we deal with the term corresponding to $j \leq J$. In this case we shall use an anisotropic version of the Taylor inequality which can be found in [17] and [24] and is recalled in [6]. It gives the existence of some $C>0$ such that

$$
\left|f_{j}(x)-P_{j, x_{0}}(x)\right| \leq C \sum_{\beta_{1}+\beta_{2} \leq k+1, \alpha_{1} \beta_{1}+\alpha_{2} \beta_{2}>s}\left|x-x_{0}\right|_{\alpha}^{\alpha_{1} \beta_{1}+\alpha_{2} \beta_{2}} \sup _{z=\left(z_{1}, z_{2}\right) \in \mathbb{R}^{2}}\left|\partial^{\beta} f_{j}\right|,
$$

with $k=\left[\max \left(s / \alpha_{1}, s / \alpha_{2}\right)\right]$. The bound (4.22) implies that there exists $C>0$ such that

$$
\begin{aligned}
& \left|f_{j}(x)-P_{j, x_{0}}(x)\right| \\
& \leq C \sum_{\substack{\beta_{1}+\beta_{2} \leq k+1 \\
\alpha_{1} \beta_{1}+\alpha_{2} \beta_{2}>s}}\left|x-x_{0}\right|_{\alpha}^{\alpha_{1} \beta_{1}+\alpha_{2} \beta_{2}} 2^{j\left(\beta_{1} \alpha_{1}+\beta_{2} \alpha_{2}\right)}\left(2^{-j s}+\left|x_{1}-a\right|^{s / \alpha_{1}}+\left|x_{2}-b\right|^{s / \alpha_{2}}\right) .
\end{aligned}
$$

Hence,

$$
\begin{aligned}
& \sum_{j \leq J}\left|f_{j}(x)-P_{j, x_{0}}(x)\right| \\
& \quad \leq C \sum_{\substack{\beta_{1}+\beta_{2} \leq k+1 \\
\alpha_{1} \beta_{1}+\alpha_{2} \beta_{2}>s}}\left|x-x_{0}\right|_{\alpha}^{\alpha_{1} \beta_{1}+\alpha_{2} \beta_{2}}\left(2^{J\left(\beta_{1} \alpha_{1}+\beta_{2} \alpha_{2}-s\right)}+2^{J\left(\beta_{1} \alpha_{1}+\beta_{2} \alpha_{2}\right)}\left|x-x_{0}\right|_{\alpha}^{s}\right) .
\end{aligned}
$$

Since $\left|x-x_{0}\right|_{\alpha} \leq 2^{-J}$ there follows

$$
\sum_{j \leq J}\left|f_{j}(x)-P_{j, x_{0}}(x)\right| \leq C\left|x-x_{0}\right|_{\alpha}^{s}
$$

Now we bound the sum $\sum_{j=J+1}^{J_{1}}\left|f_{j}(x)\right|$. By (4.21) and the definition of $J_{1}$, which depends on $J$, one has

$$
\sum_{j=J+1}^{J_{1}}\left|f_{j}(x)\right| \leq \sum_{j=J}^{J_{1}}\left(j 2^{-j s}+j\left|x-x_{0}\right|_{\alpha}^{s}\right) \leq J 2^{-J s}+J^{2}\left|x-x_{0}\right|_{\alpha}^{s} .
$$

To bound the sum $\sum_{j>J_{1}}\left|f_{j}(x)\right|$ the uniform regularity of $f$ is used, leading to

$$
\sum_{j>J_{1}}\left|f_{j}(x)\right| \leq C 2^{-J_{1} \varepsilon_{0}} \leq C 2^{-J s},
$$

the last inequality following from the definition of $J_{1}$.

Finally, by (4.22), the sum $\sum_{j>J}\left|P_{j, x_{0}}(x)\right|$ can be bounded. Indeed, for some $C>0$, one has

$$
\begin{aligned}
\sum_{j>J}\left|P_{j, x_{0}}(x)\right| & \leq \sum_{\beta_{1} \alpha_{1}+\beta_{2} \alpha_{2}<s} \frac{\left|\left(x-x_{0}\right)^{\beta}\right|}{\beta !} \sum_{j>J}\left|\partial^{\beta} f_{j}\left(x_{0}\right)\right| \\
& \leq C \sum_{\beta_{1} \alpha_{1}+\beta_{2} \alpha_{2}<s} \frac{\left|x_{1}-a\right|^{\beta_{1}}\left|x_{2}-b\right|^{\beta_{2}}}{\beta !} \sum_{j>J} 2^{j\left(\beta_{1} \alpha_{1}+\beta_{2} \alpha_{2}-s\right)} .
\end{aligned}
$$


Since $\left|x_{1}-a\right| \leq\left|x-x_{0}\right|_{\alpha}^{\alpha_{1}} \leq 2^{-J \alpha_{1}}$ and $\left|x_{2}-b\right| \leq\left|x-x_{0}\right|_{\alpha}^{\alpha_{2}} \leq 2^{-J \alpha_{2}}$ there results (4.26) $\sum_{j>J}\left|P_{j, x_{0}}(x)\right| \leq C \sum_{\beta_{1} \alpha_{1}+\beta_{2} \alpha_{2}<s} 2^{-J\left(\beta_{1} \alpha_{1}+\beta_{2} \alpha_{2}\right)} \sum_{j>J} 2^{j\left(\beta_{1} \alpha_{1}+\beta_{2} \alpha_{2}-s\right)} \leq C 2^{-J s}$.

Finally, inequalities $(4.23),(4.24),(4.25)$, and (4.26) yield that $f \in \mathcal{C}_{|\log |^{2}}^{s, \alpha}\left(x_{0}\right)$.

Theorem 3.4 is a straightforward consequence of the two-microlocal criterion and of the following lemma:

Lemma 4.11. The two following properties are equivalent:

(i) Inequality (4.18) holds.

(ii) Inequality (3.4) holds.

Proof. Assume that (4.18) holds. If $\lambda^{\prime} \subset 3 \lambda_{j_{1}, j_{2}}\left(x_{0}\right)$, then

$$
j_{1}^{\prime} \geq j_{1}, \quad j_{2}^{\prime} \geq j_{2}, \quad\left|\frac{k_{1}^{\prime}}{2^{j_{1}^{\prime}}}-a\right| \leq 2 \cdot 2^{-j_{1}^{\prime}}, \quad \text { and } \quad\left|\frac{k_{2}^{\prime}}{2^{j_{2}^{\prime}}}-b\right| \leq 2 \cdot 2^{-j_{2}^{\prime}} .
$$

Condition (4.18) implies

$$
\left|c_{\lambda^{\prime}}\right| \leq \min \left(2^{-j_{1} s / \alpha_{1}}, 2^{-j_{2} s / \alpha_{2}}\right)=2^{-\max \left(j_{1} / \alpha_{1}, j_{2} / \alpha_{2}\right) s} .
$$

Conversely, assume that (3.4) holds. Let $\lambda^{\prime}=\lambda\left(j_{1}^{\prime}, j_{2}^{\prime}, k_{1}^{\prime}, k_{2}^{\prime}\right)$ be a hyperbolic dyadic cube. Set

$$
j_{1}=\sup \left\{\ell_{1}, 2^{-j_{1}^{\prime}}+\left|\frac{k_{1}^{\prime}}{2^{j_{1}^{\prime}}}-a\right| \leq 2^{-\ell_{1}}\right\}
$$

and

$$
j_{2}=\sup \left\{\ell_{2}, 2^{-j_{2}^{\prime}}+\left|\frac{k_{2}^{\prime}}{2^{j_{2}^{\prime}}}-b\right| \leq 2^{-\ell_{2}}\right\} .
$$

We have $\lambda^{\prime} \subset 3 \lambda_{j_{1}, j_{2}}\left(x_{0}\right)$. Since (3.4) holds one has

$$
\begin{aligned}
\left|c_{\lambda^{\prime}}\right| & \leq \min \left(2^{-j_{1} s / \alpha_{1}}, 2^{-j_{2} s / \alpha_{2}}\right) \\
& \leq C \min \left(2^{-j_{1}^{\prime} s / \alpha_{1}}+\left|\frac{k_{1}^{\prime}}{2^{j_{1}^{\prime}}}-a\right|^{s / \alpha_{1}}, 2^{-j_{2}^{\prime} s / \alpha_{2}}+\left|\frac{k_{2}^{\prime}}{2^{j_{2}^{\prime}}}-b\right|^{s / \alpha_{2}}\right) .
\end{aligned}
$$

That is, (4.18) holds.

\subsection{Proof of Theorem 3.7}

The proof of Theorem 3.7 is based on the two following lemmas, analogous to Propositions 7 and 8 of [34].

Lemma 4.12. Set $\alpha=(a, 2-a)$ and define

$$
G(H, \alpha)=\left\{x \in \mathbb{R}^{2}, f \notin \mathcal{C}_{|\log |^{2}}^{H, \alpha}(x)\right\} .
$$

Let $p>0$ and $s \in(0, \omega(p, \alpha) / p]$. Then, for any $H \geq s-2 / p$,

$$
\operatorname{dim}_{H}(G(H, \alpha)) \leq H p-s p+2 .
$$

If $H<s-2 / p, \operatorname{dim}_{H}(G(H, \alpha))=-\infty$. 
Lemma 4.13. Set $\alpha=(a, 2-a)$ and define

$$
B(H, \alpha)=\left\{x \in \mathbb{R}^{2}, f \in \mathcal{C}^{H, \alpha}(x)\right\}
$$

Let $p<0$ and $s \in(0, \omega(p, \alpha) / p]$. Then

$$
\operatorname{dim}_{H}(B(H, \alpha)) \leq \operatorname{dim}_{P}(B(H, \alpha)) \leq H p-s p+2 .
$$

The proof of Lemma 4.12 in the case $H \geq s-2 / p$ is exactly the same as that of Proposition 7 of [34], except that the sets $G_{j, H}$ are replaced by the sets

$$
G(j, H, \alpha)=\left\{\lambda=\lambda\left(j_{1}, j_{2}, k_{1}, k_{2}\right),\left(j_{1}, j_{2}\right) \in \Gamma_{j}(\alpha),\left|d_{\lambda}\right| \geq 2^{-j H p}\right\} .
$$

Lemma 4.12, in the case $H<s-2 / p$, comes from the hyperbolic wavelet characterization of anisotropic Besov spaces stated in Theorem 2.6 and the Sobolev embeddings which can be proved in the anisotropic case as in the isotropic one (see [48]).

The proof of Lemma 4.13 is exactly the same as that of Proposition 8 of [34], except that the sets $B_{H}$ are replaced by the sets $B(H, \alpha)$.

Lemmas 4.12 and 4.13 then imply Theorem 3.7, since for any $\alpha=\left(\alpha_{1}, \alpha_{2}\right)$ such that $\alpha_{1}+\alpha_{2}=2$, one has

$$
E(H, \alpha) \subset\left(\bigcap_{H^{\prime}>H} G\left(H^{\prime}, \alpha\right)\right) \cap\left(\bigcup_{H^{\prime}<H} B\left(H^{\prime}, \alpha\right)\right) .
$$

Acknowledgements. We warmly thank Florent Autin and Jean Marc Freyermuth for many stimulating discussions about applications of nonparametric statistics to the analysis of anisotropic textures as well as Laurent Duval for informing us of very interesting additional references about hyperbolic wavelet analysis.

\section{References}

[1] Abry, P., Jaffard, S. And Wendt, W.: Irregularities and scaling in signal and image processing: multifractal analysis. In Benoît Mandelbrot: a life in many dimensions. World Scientific M. Frame Ed., 2012.

[2] Aimar, H. And Gómez, I.: Parabolic Besov regularity for the heat equation. Constr. Approx. 36 (2012), no. 1, 145-159.

[3] Autin, F., Claeskens, G. And Freyermuth, J-M.: Hyperbolic wavelet thresholding rules: the curse of dimensionality through the maxiset approach. Appl. Comput. Harmon. Anal. 36 (2014), no. 2, 239-255.

[4] Ayache, A.: Hausdorff dimension of the graph of the fractional Brownian sheet. Rev. Mat. Iberoamericana 20 (2004), no. 2, 395-412.

[5] Ayache, A., Roueff, F. And Xiao, Y.: Local and asymptotic properties of linear fractional stable sheets. C. R. Math. Acad. Sci. Paris 344 (2007), no. 6, 389-394.

[6] Ben Braiek, H. And Ben Slimane, M.: Baire generic anisotropic multifractal formalism in criteria in anisotropic function spaces. Preprint, 2011. 
[7] Ben Slimane, M. and Ben Braiek, H.: Directional and anisotropic regularity and irregularity criteria in Triebel wavelet bases. J. Fourier Anal. Appl. 18 (2012), no. 5, 893-914.

[8] Ben Slimane, M.: Multifractal formalism and anisotropic selfsimilar functions. Math. Proc. Cambridge Philos. Soc. 124 (1998), 329-363.

[9] Berkolă̌Ko, M.Z. And Novikov, I. YA.: Wavelet bases in spaces of differentiable functions of anisotropic smoothness. (Russian). Dokl. Akad. Nauk 323 (1992), no. 4, 615-618; translation in Russian Acad. Sci. Dokl. Math. 45 (1992), no. $2,382-386$.

[10] BerkolăKo, M. Z. and Novikov, I. Ya.: Unconditional bases in spaces of functions of anisotropic smoothness. (Russian). Trudy Mat. Inst. Steklov. 204 (1993), Issled. po Teor. Differ. Funktsii Mnogikh Peremen. i ee Prilozh. 16, 35-51; translation in Proc. Steklov Inst. Math. 1994, no. 3 (204), 27-41.

[11] Beylkin, G.: Wavelets and fast numerical algorithms. In Different perspectives on wavelets (San Antonio, TX, 1993), 89-117. Proc. Symp. Appl. Math. 47, Amer. Math. Soc., Providence, RI, 1993.

[12] Beylkin, G., Coifman, R. And Rokhlin, V.: Fast wavelet transforms and numerical algorithms. I. Comm. Pure Appl. Math. 44 (1991), no. 2, 141-183.

[13] Biermé, H., Meerschaert, M. M. and Scheffler, H. P.: Operator scaling stable random fields. Stochastic Process. Appl. 117 (2009), no. 3, 312-332.

[14] Bonami, A. And Estrade, A.: Anisotropic analysis of some Gaussian models. J. Fourier Anal. Appl. 9 (2003), 215-236.

[15] Bownik, M.: Atomic and molecular decomposition of anisotropic Besov spaces. Math. Z. 250 (2005), 539-571.

[16] Bownik, M. And Ho, K. P.: Atomic and molecular decomposition of anisotropic Triebel-Lizorkin spaces. Trans. Amer. Math. Soc. 358 (2006), no. 4, 1469-1510.

[17] Calderón, A.P. And Torchinsky, A.: Parabolic maximal functions associated with a distribution. II. Advances in Math. 24 (1977), 101-171.

[18] Candès, E. And Demanet, L.: The curvelet representation of wave propagators is optimally sparse. Comm. Pure Appl. Math. 58 (2005), no. 11, 1472-1528.

[19] Clausel, M. and Vedel, B.: An optimality result about sample paths properties of operator scaling Gaussian random fields. Ann. Univ. Buchar. Math. Ser. 4 (LXII) (2013), 375-409.

[20] Davies, S. And Hall, P.: Fractal analysis of surface roughness by using spatial data. J. R. Stat. Soc. Ser. B Stat. Methodol. 61 (1999), 3-37.

[21] DeVore, R. A., Konyagin, S. V. and Temlyakov, V. N.: Hyperbolic wavelet approximation. Constr. Approx. 14 (1998), 1-26.

[22] Donoho, D.: Wedgelets: nearly minimax estimation of edges. Ann. Statist. 27 (1999), no. 3, 859-897.

[23] FAlConer, K.: Fractal geometry. Mathematical foundations and applications. Wiley \& Sons, 1990.

[24] Folland, G. B. And Stein, E. M.: Hardy spaces on homogeneous groups. Mathematical Notes 28, Princeton University Press, University of Tokyo Press, 1982.

[25] Frazier, M. And Jawerth,B.: Decomposition of Besov spaces. Indiana Univ. Math. J. 34 (1985), no. 4, 777-799. 
[26] Garrigós, G. and Tabacco, A.: Wavelet decompositions of anisotropic Besov spaces. Math. Nachr. 239/240 (2002), 80-102.

[27] Guo, K. And Labate, D.: Analysis and detection of surface discontinuities using the 3D continuous shearlet transform. Appl. Comput. Harmon. Anal. 30 (2011), no. 2, 231-242.

[28] Guo, K. And Labate, D.: Representation of Fourier integral operators using shearlets. J. Fourier Anal. Appl. 14 (2008), no. 3, 327-371.

[29] Haroske, D. and TAmási, E.: Wavelet frames for distributions in anisotropic Besov spaces. Georgian Math. J. 12 (2005), no. 4, 637-658.

[30] Hochmuth, R.: Wavelet characterizations for anisotropic Besov spaces. Appl. Comput. Harmon. Anal. 12 (2002), 179-208.

[31] Hоснмuтн, R.: $n$-term approximation in anisotropic function spaces. Math. Nachr. 244 (2002), 131-149.

[32] Jacques, L., Duval, L., Chaux, C. And Peyré, G.: A panorama on multiscale geometric representations, intertwining spatial, directional and frequency selectivity. Signal Processing 91 (2011), 2699-2730.

[33] Jaffard, S.: Pointwise and directional regularity of nonharmonic Fourier series. Appl. Comput. Harmon. Anal. 28 (2010), no. 3, 251-266.

[34] JAFFARD, S.: Wavelet techniques in multifractal analysis. In Fractal geometry and applications: a jubilee of Benoit Mandelbrot, Part 2, 91-151. Proc. Sympos. Pure Math. 72, Part 2, Amer. Math. Soc., Providence, RI, 2004.

[35] Lakhonchai, P., Sampo, J. and Sumetkijakan, S.: Shearlet transforms and Hölder regularities. To appear in Int. J. Wavelets Multiresolut. Inf. Process.

[36] Meyer, Y.: Ondelettes et opérateurs. I. Actualités Mathématiques, Hermann, Paris, 1990.

[37] Neumann, M. H.: Multivariate wavelet thresholding in anisotropic function spaces. Statist. Sinica 10 (2000), no. 2, 399-431.

[38] Neumann, M. H. And von Sachs, R.: Wavelet thresholding in anisotropic function classes and application to adaptative estimation of evolutionary spectra. Ann. Statist. 25 (1997), 38-76.

[39] Nualtong, K. And Sumetkijakan, S.: Analysis of Hölder regularities by waveletlike transforms with parabolic scaling. Thai J. Math. 3 (2005), no. 2, 275-283.

[40] PARisi, G. AND Frisch, U.: On the singularity spectrum of fully developped turbulence. In Turbulence and predictability in geophysical fluid dynamics, 84-87. Proceedings of the International Summer School in Physics Enrico Fermi, North Holland, 1985.

[41] Ponson, L., Bonamy, D., Auradou, H., Mourot, G., Morel, S., BouChaud, E., Guillot, C. And Hulin, J.P.: Anisotropic self-affine properties of experimental fracture surfaces. Int. J. Fracture 140 (2006), 27-37.

[42] Roux, S. G., Clausel, M., Vedel, B., Jaffard, S. and Abry, P.: Self-similar anisotropic texture analysis: the hyperbolic wavelet transform contribution. IEEE Trans. Image Process. 22 (2013), no. 11, 4353-4363.

[43] Sampo, J. And Sumetkijakan, S.: Estimations of Hölder regularities and direction of singularity by Hart Smith and curvelet transforms. J. Fourier Anal. Appl. 15 (2009), no. 1, 58-79. 
[44] Schmeisser, H. J. And Sickel, W.: Spaces of functions of mixed smoothness and approximation from hyperbolic crosses. J. Approx. Theory 128 (2004), 115-150.

[45] Sickel, W. And Ullrich, T.: Tensor products of Sobolev-Besov spaces and applications to approximation from the hyperbolic cross. J. Approx. Theory 161 (2009), no. $2,748-786$.

[46] Smith, H.: A Hardy space for Fourier integral operators. J. Geom. Anal. 8 (1998), no. 4, 629-653.

[47] TaO, T. And Vargas, A.: A bilinear approach to cone multipliers. II. Applications. Geom. Funct. Anal. 10 (2000), no. 1, 216-258.

[48] Triebel, H.: Theory of function spaces, III. Monographs in Mathematics 100, Birhäuser Verlag, Basel, 2006.

[49] Triebel, H.: Wavelet bases in anisotropic function spaces. In Function spaces, differential operators and nonlinear analysis, (FSDONA-04, Milovy, Czech Republic, 2004), 370-387. Math. Inst. Acad. Sci. Czech Republic, Praha, 2005.

[50] Triebel, H.: Interpolation theory, function spaces, differential operators. NorthHolland Mathematical Library 18, Amsterdam-New York, 1978.

[51] Westerink, P.H.: Subband coding of images. PhD Thesis, Delft University of Technology, Delft, 1989.

[52] Yamazaki, M.: A quasihomogeneous version of paradifferential operators. I. Boundedness on spaces of Besov type. J. Fac. Sci. Univ. Tokyo Sect. IA Math. 33 (1986), no. 1, 131-174.

[53] Yamazaki, M.: A quasihomogeneous version of paradifferential operators. II. A symbol calculus. J. Fac. Sci. Univ. Tokyo Sect. IA Math. 33 (1986), no. 2, 311-345.

Received November 22, 2013.

PAtrice Abry: Physics Dept., ENS Lyon, CNRS, UMR5672, Lyon, France.

E-mail: patrice.abry@ens-lyon.fr

Marianne Clausel: University of Grenoble, CNRS, Laboratoire Jean Kuntzmann UMR 5224, Saint Martin d'Hères, France.

E-mail: Marianne.Clausel@imag.fr

StÉphane Jaffard: Université Paris Est, LAMA, UMR 8050, Créteil, France.

E-mail: stephane.jaffard@u-pec.fr

Stéphane G. Roux: Physics Dept., ENS Lyon, CNRS, UMR5672, Lyon, France.

E-mail: stephane.roux@ens-lyon.fr

BÉAtrice Vedel: LMBA, Université de Bretagne Sud, European University of Bretagne, Vannes, France.

E-mail: beatrice.vedel@univ-ubs.fr

This work was supported by the ANR grant AMATIS (ANR2011 BS01011 02) and the CNRS Groupe de Recherche Analyse Multifractale. 